\title{
The fundamental group and Betti numbers of toric origami manifolds
}

\author{
TARA S HOLM \\ ANA Rita PIRES
}

\begin{abstract}
Toric origami manifolds are characterized by origami templates, which are combinatorial models built by gluing polytopes together along facets. In this paper, we examine the topology of orientable toric origami manifolds with coorientable folding hypersurface. We determine the fundamental group. In our previous paper, we studied the ordinary and equivariant cohomology rings of simply connected toric origami manifolds. We conclude this paper by computing some Betti numbers and cohomology rings in the non-simply connected case.
\end{abstract}

53D20; 55N91, 57R91

\section{Introduction}

Smooth toric varieties and their generalizations are manifolds whose geometry and topology can be characterized by combinatorial models. The interplay between geometry and topology on the one hand and algebra, combinatorics, and discrete geometry on the other has been integral to our understanding of toric varieties. In this paper, we study toric origami manifolds, a class of toric manifolds that arise in symplectic geometry. The geometry of toric origami manifolds is encoded in an origami template: a collection of (equi-dimensional) polytopes with certain facets identified. In our previous paper [13], we studied the simplest examples of toric origami manifolds, the acyclic ones. In this manuscript, we develop new techniques to address the complications that arise in the cyclic case.

We first study the fundamental group of a toric origami manifold. Building on work of Masuda and Park [17] and others, we use the combinatorics of the origami template to determine the fundamental group of a toric origami manifold (Theorem 2-14). The key trick is to build a simply connected cover of the origami template. As a consequence of our result, we may deduce that a toric origami manifold is simply connected if and only if it is acyclic. We can use our result (namely, the form of the fundamental group) to show the existence of a 4-dimensional manifold equipped with an effective $\mathbb{T}^{2}$ action which is not a toric origami manifold (Remark 2-18). 
We then turn to the Betti numbers of a toric origami manifold. When $M$ is orientable, there is a natural decomposition $M=M_{+} \cup M_{-}$, where $M_{+} \cap M_{-} \cong Z$ is the folding hypersurface. There are situations in which we have control of the cohomology groups of $Z$ and of $M_{+} \sqcup M_{-} \cong M \backslash Z$. We may then use a Mayer-Vietoris argument to determine certain Betti numbers of $M$. In dimension 4, we may determine all Betti numbers, and hence the Euler characteristic (Theorem 5-2). Again, this allows us to rule out a possible toric origami structure on a specific 4-manifold which is known to admit an effective $\mathbb{T}^{2}$ action (Remark 5-3).

The results in this paper were developed simultaneously to those in the recent preprint of Ayzenberg, Masuda, Park and Zeng [2]. Their techniques rely on the assumption that proper faces of the orbit space are acyclic. With this hypothesis, the authors are, for the most part, able to determine the ring structure in cohomology, in terms of equivariant cohomology. Our results apply to all origami templates, but our cohomological results are only about Betti numbers.

The remainder of the paper is organized as follows. We outline the basic notions and notation in Section 1. We compute the fundamental group of a toric origami manifold in Section 2. In Section 3, we establish certain Betti numbers of components of $M \backslash Z$, and in Section 4, we describe a method to compute the Betti numbers of $Z$. We then use those calculations in Section 5 to determine some of the Betti numbers of toric origami manifolds. In particular, we determine all of the Betti numbers of a 4-dimensional toric origami manifold, in terms of combinatorial data from the origami template. We conclude with the full details of an example in 6 dimensions, showing how our techniques are tractable even in higher dimensions, when faced with specific examples.

Acknowledgements Tara Holm was partially supported by grant number 208975 from the Simons Foundation and NSF grant DMS-1206466. Both authors were partially supported by the National Science Foundation under agreement number DMS-1128155. Any opinions, findings and conclusions or recommendations expressed in this material are those of the authors and do not necessarily reflect the views of the National Science Foundation.

We would like to thank Strom Borman, Allen Hatcher, Allen Knutson, Nick Sheridan and Reyer Sjamaar for useful conversations, as well as the anonymous referee for helpful comments.

Remarks 2-18 and 5-3 resulted from discussions of the second author with Ana Cannas da Silva, and we are grateful for the possibility to include them here. Both authors are grateful for the hospitality of the Oxford Mathematical Institute and the Institute for Advanced Study during the preparation of this paper. 


\section{Origami manifolds}

This is a summary of the background and set-up described in our previous paper [13, Section 2], where there are more examples and details. We include it again here to set the notation. There is one new item: toric origami manifolds with boundary, which are an ingredient in Section 2.

\subsection{Symplectic manifolds}

We begin with a very quick review of symplectic geometry following [4]. Let $M$ be a manifold equipped with a symplectic form $\omega \in \Omega^{2}(M)$; that is, $\omega$ is closed $(d \omega=0)$ and non-degenerate. In particular, the non-degeneracy condition implies that $M$ must be an even-dimensional manifold.

Suppose that a compact connected abelian Lie group $\mathbb{T}=\left(\mathbb{S}^{1}\right)^{n}$ acts on $M$ preserving $\omega$. The action is weakly Hamiltonian if for every vector $\xi \in \mathfrak{t}$ in the Lie algebra $\mathfrak{t}$ of $\mathbb{T}$, the vector field

$$
\mathcal{X}_{\xi}(p)=\left.\frac{d}{d t}[\exp (t \xi) \cdot p]\right|_{t=0}
$$

is a Hamiltonian vector field. That is, we require $\omega\left(\mathcal{X}_{\xi}, \cdot\right)$ to be an exact one-form: ${ }^{1}$

$$
\omega\left(\mathcal{X}_{\xi}, \cdot\right)=d \phi^{\xi} \text {. }
$$

Thus each $\phi^{\xi}$ is a smooth function on $M$ defined by the differential equation (1-1), so determined up to a constant. Taking them together, we may define a moment map

$$
\Phi: M \rightarrow \mathfrak{t}^{*}, \quad p \mapsto\left(\begin{array}{llc}
\mathfrak{t} & \rightarrow & \mathbb{R} \\
\xi & \mapsto \phi^{\xi}(p)
\end{array}\right) .
$$

The action is Hamiltonian if the moment map $\Phi$ can be chosen to be a $\mathbb{T}$-invariant map. Atiyah [1] and Guillemin and Sternberg [11] have shown that when $M$ is a compact Hamiltonian $\mathbb{T}$-manifold, the image $\Phi(M)$ is a convex polytope, and is the convex hull of the images of the fixed points $\Phi\left(M^{\mathbb{T}}\right)$.

For an effective $e^{2}$ Hamiltonian $\mathbb{T}$ action on $M$, $\operatorname{dim}(\mathbb{T}) \leq \frac{1}{2} \operatorname{dim}(M)$. We say that the action is toric if this inequality is in fact an equality. A symplectic manifold $M$ with a toric Hamiltonian $\mathbb{T}$ action is called a symplectic toric manifold. Delzant used the moment polytope to classify symplectic toric manifolds.

A polytope $\Delta$ in $\mathbb{R}^{n}$ is simple if there are $n$ edges incident to each vertex, and it is rational if each edge vector has rational slope: it lies in $\mathbb{Q}^{n} \subset \mathbb{R}^{n}$. A simple polytope is

\footnotetext{
${ }^{1}$ The one-form $\omega\left(\mathcal{X}_{\xi}, \cdot\right)$ is automatically closed because the action preserves $\omega$.

${ }^{2} \mathrm{An}$ action is effective if no non-trivial subgroup acts trivially.
} 
smooth at a vertex if the $n$ primitive vectors parallel to the edges at the vertex span the lattice $\mathbb{Z}^{n} \subseteq \mathbb{R}^{n}$ over $\mathbb{Z}$. It is smooth if it is smooth at each vertex. A simple rational smooth convex polytope is called a Delzant polytope. We may now state Delzant's result.

\section{Theorem 1-2 (Delzant [8]) There is a one-to-one correspondence}

$\{$ compact toric symplectic manifolds $\} \leftrightarrow \rightsquigarrow\{$ Delzant polytopes $\}$,

up to equivariant symplectomorphism on the left-hand side and affine equivalence on the right-hand side.

\subsection{Origami manifolds}

We now relax the non-degeneracy condition on $\omega$ following [5]. A folded symplectic form on a $2 n$-dimensional manifold $M$ is a 2 -form $\omega \in \Omega^{2}(M)$ that is closed $(d \omega=0)$, whose top power $\omega^{n}$ intersects the zero section transversely on a subset $Z$ and whose restriction to points in $Z$ has maximal rank. The transversality forces $Z$ to be a codimension-1 embedded submanifold of $M$. We call $Z$ the folding hypersurface or fold.

Let $i: Z \hookrightarrow M$ be the inclusion of $Z$ as a submanifold of $M$. Our assumptions imply that $i^{*} \omega$ has a 1 -dimensional kernel on $Z$. This line field is called the null foliation on $Z$. An origami manifold is a folded symplectic manifold $(M, \omega)$ whose null foliation is fibrating: $\pi: Z \rightarrow B$ is a fiber bundle with orientable circle fibers over a compact base $B$. The form $\omega$ is called an origami form and the bundle $\pi$ is called the null fibration. A diffeomorphism between two origami manifolds which intertwines the origami forms is called an origami-symplectomorphism. The definition of a Hamiltonian action only depends on $\omega$ being closed. Thus, in the folded framework, we may define moment maps and toric actions exactly as in Section 1.1.

An oriented origami manifold $M$ with fold $Z$ may be unfolded into a symplectic manifold as follows. Consider the closures of the connected components of $M \backslash Z$, a manifold with boundary which consists of two copies of $Z$. We collapse the fibers of the null fibration by identifying the boundary points that are in the same fiber of the null fibration of each individual copy of $Z$. The result, $M_{0}:=(M \backslash Z) \cup B_{1} \cup B_{2}$, is a (disconnected) smooth manifold that can be naturally endowed with a symplectic form which on $M_{0} \backslash\left(B_{1} \cup B_{2}\right)$ coincides with the origami form on $M \backslash Z$. Because this can be achieved using symplectic cutting techniques, the resulting manifold $M_{0}$ is called the symplectic cut space (and its connected components the symplectic cut pieces), and the process is also called cutting. The symplectic cut space of a nonorientable origami manifold is the $\mathbb{Z}_{2}$-quotient of the symplectic cut space of its orientable double cover. 
The cut space $M_{0}$ of an oriented origami manifold $(M, \omega)$ inherits a natural orientation. It is the orientation on $M_{0}$ induced from the orientation on $M$ that matches the symplectic orientation on the symplectic cut pieces corresponding to the subset of $M \backslash Z$ where $\omega^{n}>0$ and the opposite orientation on those pieces where $\omega^{n}<0$. In this way, we can associate a + or - sign to each of the symplectic cut pieces of an oriented origami manifold, as well as to the corresponding connected components of $M \backslash Z$.

Remark 1-3 In this paper we restrict to origami manifolds whose fold is coorientable; that is, the fold has an orientable neighborhood. Note that this does not imply that the manifold is orientable. Indeed, for an orientable $M$, the condition that $\omega^{n}$ intersects the zero section transversally implies that the connected components of $M \backslash Z$ which are adjacent in $M$ have opposite signs. Since $M$ is connected, picking a sign for one connected component of $M \backslash Z$ determines the signs for all other components. As a consequence, an origami manifold $M$ with coorientable fold is orientable if and only if it is possible to make such a global choice of signs for the connected components of $M \backslash Z$.

Proposition 1-4 [5, Propositions 2.5 and 2.7] Let $M$ be a (possibly disconnected) symplectic manifold with a codimension two symplectic submanifold $B$ and a symplectic involution $\gamma$ of a tubular neighborhood $\mathcal{U}$ of $B$ which preserves $B .^{3}$ Then there is an origami manifold $\tilde{M}$ such that $M$ is the symplectic cut space of $\tilde{M}$. Moreover, this manifold is unique up to origami-symplectomorphism.

This newly created fold $Z \subset \tilde{M}$ involves the radial projectivized normal bundle of $B \subset M$, so we call the origami manifold $\tilde{M}$ the radial blow-up of $M$ through $(\gamma, B)$. The cutting operation and the radial blow-up operation are in the following sense inverses of each other.

Proposition 1-5 [5, Proposition 2.37] Let $M$ be an origami manifold with cut space $M_{0}$. The radial blow-up $\tilde{M}_{0}$ is origami-symplectomorphic to $M$.

There exist Hamiltonian versions of these two operations which may be used to see that the moment map $\Phi$ for an origami manifold $M$ coincides, on each connected component of $M \backslash Z$ with the induced moment map $\Phi_{i}$ on the corresponding symplectic cut piece $M_{i}$. As a result, the moment image $\Phi(M)$ is the union of convex polytopes $\Delta_{i}$.

\footnotetext{
${ }^{3}$ In the noncoorientable case, the involution must satisfy additional conditions; see [5, Definition 2.23]. In the coorientable case, we have $B=B_{1} \cup B_{2}$ and the involution $\gamma$ maps a tubular neighborhood of $B_{1}$ to one of $B_{2}$ and vice versa.
} 
Furthermore, if the circle fibers of the null fibration for a connected component $\mathcal{Z}$ of the fold $Z$ are orbits for a circle subgroup $\mathbb{S}^{1} \subset \mathbb{T}$, then $\Phi(\mathcal{Z})$ is a facet of each of the two polytopes corresponding to neighboring components of $M \backslash Z$. Let us denote these two polytopes $\Delta_{1}$ and $\Delta_{2}$. We note that they must agree near $\Phi(\mathcal{Z})$ : there is a neighborhood $\mathcal{V}$ of $\Phi(\mathcal{Z})$ in $\mathbb{R}^{n}$ such that $\Delta_{1} \cap \mathcal{V}=\Delta_{2} \cap \mathcal{V}$. The condition that the circle fibers are orbits is automatically satisfied when the action is toric, and in that case there is a classification theorem in terms of the moment data.

The moment data of a toric origami manifold can be encoded in the form of an origami template, originally defined in [5, Definition 3.12]. Definition 1-6 below is a refinement of that original definition. Following [10, page 5], a graph $G$ consists of a nonempty set $V$ of vertices and a set $E$ of edges together with an incidence relation that associates an edge with its two end vertices, which need not be distinct. Note that this allows for the existence of (distinguishable) multiple edges with the same two end vertices, and of loops whose two end vertices are equal. The degree of a vertex $v \in V$ in a graph $G$ is equal to the number non-loop edges incident to $v$ plus twice the number of loops incident to $v$.

We introduce some additional notation: let $\mathcal{D}_{n}$ be the set of all Delzant polytopes in $\mathbb{R}^{n}$ and $\mathcal{E}_{n}$ the set of all subsets of $\mathbb{R}^{n}$ which are facets of elements of $\mathcal{D}_{n}$.

Definition 1-6 An $n$-dimensional origami template consists of a graph $G$, called the template graph, and a pair of maps $\Psi_{V}: V \rightarrow \mathcal{D}_{n}$ and $\Psi_{E}: E \rightarrow \mathcal{E}_{n}$ such that:

(1) If $e$ is an edge of $G$ with end vertices $u$ and $v$, then $\Psi_{E}(e)$ is a facet of each of the polytopes $\Psi_{V}(u)$ and $\Psi_{V}(v)$, and these polytopes agree near $\Psi_{E}(e)$.

(2) If $v$ is an end vertex of each of the two distinct edges $e$ and $f$, then $\Psi_{E}(e) \cap$ $\Psi_{E}(f)=\varnothing$.

The polytopes in the image of the map $\Psi_{V}$ are the Delzant polytopes of the symplectic cut pieces. For each edge $e$, the set $\Psi_{E}(e)$ is a facet of the polytope(s) corresponding to the end vertices of $e$. We refer to such a set as a fold facet, as it is the image of the connected components of the folding hypersurface. ${ }^{4}$

With these combinatorial data in place, we may now state the classification theorem.

\section{Theorem 1-7 [5, Theorem 3.13] There is a one-to-one correspondence}

$\{$ compact toric origami manifolds\} «

up to equivariant origami-symplectomorphism on the left-hand side, and affine equivalence of the image of the template in $\mathbb{R}^{n}$ on the right-hand side.

\footnotetext{
${ }^{4}$ A noncoorientable connected component of the folding hypersurface corresponds to a loop edge $e$.
} 
For the purposes of this paper, we need to work with toric origami manifolds with a certain type of boundary. We begin by defining templates with boundary. To do so, we now allow our graph $G$ to have dangling edges, that is, an edge that has only one endpoint. Note that this is different from a loop edge. In particular, for the purpose of computing the degree of a vertex, a dangling edge counts as a non-loop edge.

Definition 1-8 An n-dimensional origami template with boundary consists of a graph $G$, possibly including dangling edges, called the template graph, and a pair of maps $\Psi_{V}: V \rightarrow \mathcal{D}_{n}$ and $\Psi_{E}: E \rightarrow \mathcal{E}_{n}$ satisfying the conditions (1) and (2) in Definition 1-6.

Remark 1-9 Note that condition (1) of Definition 1-6 does not impose a constraint on a dangling edge, but condition (2) may do so.

To define the toric origami manifold with boundary associated to an origami template with boundary, we may use a construction motivated by Theorem 1-7. In this way, the boundary of the origami manifold is contained in the fold. More specifically, the component of the boundary corresponding to the dangling edge $e$ is a principal circle bundle over the toric symplectic manifold with moment image $\Psi_{E}(e)$. If we collapse the circle fibers of this fibration, we obtain a toric origami manifold, possibly with boundary, with the dangling edge $e$ removed from the template graph.

This is not the most general definition of a toric origami manifold with boundary, but it is the version that we will need in the remainder of the paper.

The orbit space $X=M / \mathbb{T}$ of a toric origami manifold, possibly with boundary, is closely related to the origami template. When $M$ is a toric symplectic manifold, then the orbit space may be identified with the corresponding Delzant polytope; this identification is achieved by the moment map. For a toric origami manifold, possibly with boundary, the orbit space is realized as the topological space obtained by gluing the polytopes in $\Psi_{V}(V)$ along the fold facets as specified by the map $\Psi_{E}$. More precisely, the orbit space is the quotient

$$
X=\bigsqcup_{v \in V}\left(v, \Psi_{V}(v)\right) / \sim,
$$

where we identify $(u, x) \sim(v, y)$ if there exists an edge $e$ with endpoints $u$ and $v$ and the points $x=y \in \Psi_{E}(e) \subset \mathbb{R}^{n}$. Again, this identification is achieved by the moment map. In simple low-dimensional examples, we can visualize the orbit space by superimposing the polytopes $\Psi_{V}(v)$ in $\mathbb{R}^{n}$ and indicating which of their facets to identify. There is a deformation retraction from orbit space $X$ to the template graph. 
There is a natural description of the faces of $X$. The facets of a polytope are wellunderstood. The set of facets of $X$ is

$$
\begin{aligned}
& \bigsqcup_{v \in V}(v, F) / \sim, \\
& F \text { facet of } \Psi_{V}(v) \\
& F \text { not a fold facet }
\end{aligned}
$$

where the equivalence relation is induced by the one in (1-10). The faces of $X$ are non-empty intersections of facets in $X$, together with $X$ itself. This notion of face of the orbit space agrees with Masuda and Panov's definition [16, Section 4.1].

\section{The fundamental group of toric origami manifolds}

We now proceed to compute the fundamental group of a toric origami manifold $M$. As our manifolds are always connected, we suppress the notation of a basepoint. Key to this calculation are two lattices that arise in the definition of $M$ by its origami template.

Definition 2-1 The Delzant polytopes are subsets of $\mathbb{R}^{n}$, and the Delzant condition refers to a fixed choice of lattice $N=\mathbb{Z}^{n} \subset \mathbb{R}^{n}$. An important sublattice of $N$ is $N_{X}$, the sublattice spanned (over $\mathbb{Z}$ ) by the normal vectors to the facets of $X=M / \mathbb{T}$.

Masuda and Park have investigated the relationship between the fundamental group of $M$, that of $X$, and $N / N_{X}$.

Proposition 2-2 [17, Proposition 3.4] Let $M$ be an orientable toric origami manifold and let $N_{X}$ be as in Definition 2-1. Let $q_{*}: \pi_{1}(M) \rightarrow \pi_{1}(X)$ be the homomorphism induced from the quotient map $q: M \rightarrow X$. Then there is an epimorphism

$$
\rho: N / N_{X} \times \pi_{1}(X) \rightarrow \pi_{1}(M)
$$

such that the composition $q_{*} \circ \rho: N / N_{X} \times \pi_{1}(X) \rightarrow \pi_{1}(X)$ is the projection on the second factor, in particular, $\operatorname{ker}(\rho)$ is contained in $N / N_{X}$.

Remark 2-3 As Masuda and Park note, $N / N_{X}$ is trivial, finite cyclic or infinite cyclic. When $N / N_{X}$ is trivial, then $\rho$ is an isomorphism.

We aim to show that $\rho$ is an isomorphism. We now introduce several auxiliary spaces that will allow us to identify $\pi_{1}(M)$. Let $\tilde{X}$ denote the universal cover of the orbit space $X$. Let $\tilde{M}$ be the toric origami manifold corresponding to $\tilde{X}$. Note that $\tilde{M}$ is non-compact unless the original template graph is a tree. Then there is a covering map $V: \tilde{M} \rightarrow M$ and an injection $V_{*}: \pi_{1}(\tilde{M}) \rightarrow \pi_{1}(M)$. 
Choose a fundamental domain $\mathcal{D}$ for the action of the deck transformations on $\tilde{X}$ and consider its closure $\tilde{X}_{0}=\overline{\mathcal{D}}$ inside $\tilde{X}$. This has template graph $\widetilde{G}_{0}$, a spanning tree of the original template graph $G$ for $M$, together with some extra dangling edges. More explicitly, for every edge $e$ in $G$ that is not in the spanning tree, there are now two dangling edges in $\widetilde{G}_{0}$, one emanating from each end vertex of $e$. The manifold $\tilde{M}_{0}$ is an origami manifold with boundary. Note that in the same way that $X$ can be recovered from $\tilde{X}_{0}$ by gluing along some of the facets, and $G$ may be recovered from $\widetilde{G}_{0}$ by splicing the dangling edges described above, the origami manifold $M$ can be recovered from $\tilde{M}_{0}$ by appropriately identifying boundary components to each other. Recall that the fundamental group of $X$ is a free group $F_{\ell}$, since $X$ deformation retracts to the template graph. The Cayley graph of the free group $F_{\ell}$ is an infinite regular tree of degree $2 \ell$. We may think of $\tilde{X}$ in terms of this infinite tree, where each vertex represents a copy of $\widetilde{X}_{0}$ and the edges represent the facets by which the copies of $\tilde{X}_{0}$ are glued together. We introduce auxiliary spaces $\tilde{X}_{i}$, for $i \geq 0$, which consist of the $\left((2 \ell)^{i+1}-1\right) /(2 \ell-1)$ copies of $\tilde{X}_{0}$ that are distance at most $i$ from the identity copy of $\tilde{X}_{0}$ in the Cayley graph of $F_{\ell}$. We then may define the origami manifold with boundary $\tilde{M}_{i}$ to have template with boundary $\tilde{X}_{i}$.

We note that the spaces $\tilde{X}_{i}$ and the spaces $\tilde{M}_{i}$ are nested. That is, we have a commutative diagram

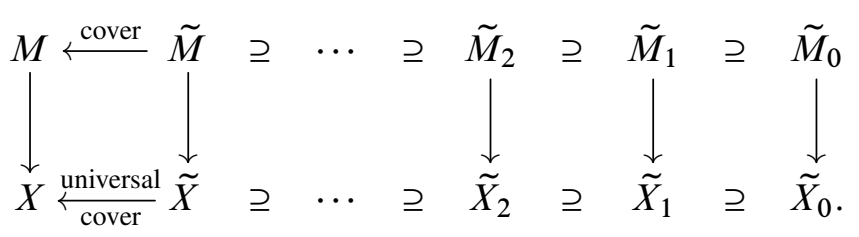

We also include Figure 2.4 below showing $X, \tilde{X}_{0}, \tilde{X}_{1}$ and $\tilde{X}_{2}$.

We now wish to compute the fundamental groups $\pi_{1}\left(\tilde{M}_{i}\right)$ and $\pi_{1}(\tilde{M})$. Danilov computed the fundamental group of a normal toric variety associated to a fan $[7$, Proposition 9.3]; a detailed proof is given in [6, Theorem 12.1.10].

Theorem 2-5 Let $\Sigma$ be a fan in $N_{\mathbb{R}}$ and let $N_{\Sigma}$ be the sublattice of $N$ generated by $|\Sigma| \cap N$. Then the fundamental group of the normal toric variety $X_{\Sigma}$ is $\pi_{1}\left(X_{\Sigma}\right) \cong$ $N / N_{\Sigma}$.

We begin by computing $\pi_{1}\left(\tilde{M}_{i}\right)$.

Lemma 2-6 Let $M$ be an origami manifold, possibly with boundary, such that the orbit space $X$ is simply connected (or equivalently, the template graph $G$ of $M$ is a tree). Let $N_{X}$ be the sublattice of $N$ generated by the rays of the multi-fan corresponding to $M$. Then the fundamental group $\pi_{1}(M) \cong N / N_{X}$. 

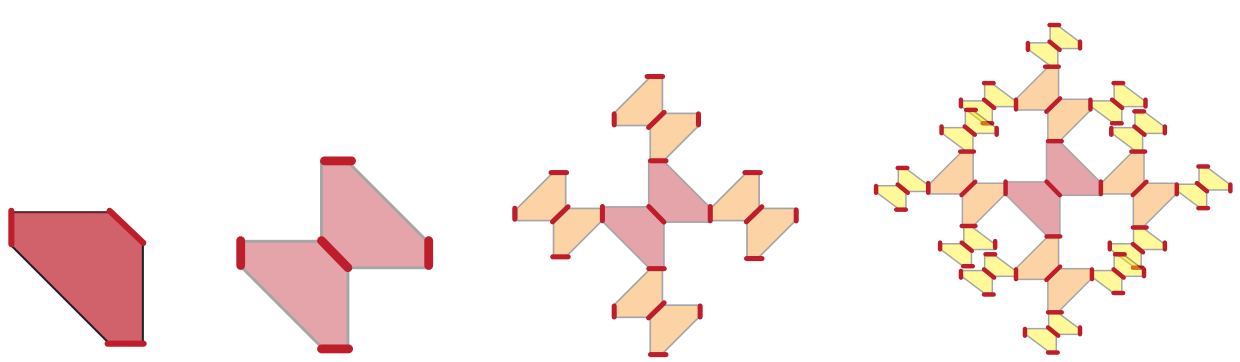

Figure 2.4: From left to right: The moment map image of a toric origami manifold $M$ whose template graph has two vertices and three edges (the polytopes corresponding to the two vertices are identical and appear superimposed); and representations of $\tilde{X}_{0}, \tilde{X}_{1}$ and $\tilde{X}_{2}$, drawn unfolded and with shrinking polytopes to prevent too many overlaps. The red boundary facets correspond to the dangling edges of the template graph. The moment images of each of these 3 spaces looks like the leftmost figure, but their templates are all different.

Proof We proceed by induction on the number of vertices in the template graph $G$. The base case is when there is a single vertex. Then the manifold $M$ is a symplectic manifold (possibly with boundary), and the corresponding multi-fan is in fact a fan $\Sigma$. Then $M$ is homeomorphic to the normal toric variety $X_{\Sigma}$, and the result is a direct application of Theorem 2-5.

For the induction step, we pick a leaf vertex $v$ of $G$. Denote the vertex set of $G$ by $V$ and the edge set $E$. Given the leaf vertex $v$, let $e$ be the edge that connects it to the rest of $G$ and $f_{1}, f_{2}, \ldots$ : the (possibly empty) list of dangling edges emanating from $v$. Let $\operatorname{Star}(v)$ be the graph with a single vertex $v$ and dangling edges $\tilde{e}, f_{1}, f_{2}, \ldots$, where $\tilde{e}$ is the new dangling edge obtained from $e$. Next, let $\operatorname{Star}(V \backslash v)$ be the graph with vertex set $V \backslash\{v\}$ and edge set $E \backslash\left\{e, f_{1}, f_{2}, \ldots\right\} \cup\{\tilde{e}\}$, where $\tilde{e}$ is the new dangling edge obtained from $e$.

We now describe a cover of $M$ with two open sets. The first set, $A$, is a small neighborhood in $M$ of the toric origami manifold with boundary $M_{1}$ whose template graph is $\operatorname{Star}(v)$ and orbit space is $X_{1}$. We may choose $A$ so that it deformation retracts to $M_{1}$. By Theorem $2-5, \pi_{1}(A) \cong N / N_{X_{1}}$. The second set, $B$, is a small neighborhood in $M$ of the toric origami manifold with boundary $M_{2}$ whose template graph is $\operatorname{Star}(V \backslash v)$ and orbit space is $X_{2}$. We may choose $B$ so that it deformation retracts to $M_{2}$. By the induction hypothesis, $\pi_{1}(B) \cong N / N_{X_{2}}$.

We note that the intersection $A \cap B$ is the tubular neighborhood of the connected component $\mathcal{Z}$ of the fold $Z$ corresponding to the edge $e$. It is homeomorphic to the 
toric variety whose fan has rays which are the normals to the facets in the polytope $\Psi_{V}(v)$ that are adjacent to the fold facet $\Psi_{E}(e)$. Thus, we may apply Theorem 2-5 to deduce that $\pi_{1}(A \cap B) \cong N / N_{A \cap B}$, where $N_{A \cap B}$ is the sublattice of $N$ spanned by the rays described in the previous sentence.

We may apply the Seifert-van Kampen theorem to deduce that

$$
\pi_{1}(M) \cong N / N_{X_{1}} * N / N_{A \cap B} N / N_{X_{2}} .
$$

As in [6, proof of Theorem 12.1.10], the final step is to use presentations of the groups $N / N_{X_{1}}, N / N_{X_{2}}$ and $N / N_{A \cap B}$ in terms of generators and relations to conclude that

$$
N / N_{X_{1}} * N / N_{A \cap B} N / N_{X_{2}}=N /\left(N_{X_{1}}+N_{X_{2}}\right)=N / N_{X} .
$$

This completes the proof.

We may now compute $\pi_{1}\left(\tilde{M}_{i}\right)$, then subsequently $\pi_{1}(\tilde{M})$.

Corollary 2-7 Let $\tilde{M}_{i}$ be the origami manifold with boundary with orbit space $\tilde{X}_{i}$, as described above. For each $i \geq 0$, the fundamental group is $\pi_{1}\left(\widetilde{M}_{i}\right) \cong N / N_{\tilde{X}_{i}}=N / N_{X}$.

Proof The only missing ingredient is to notice that $N_{\tilde{X}_{i}}=N_{X}$ for each $i$.

Corollary 2-8 Let $\tilde{M}$ be the toric origami manifold with boundary with orbit space $\tilde{X}$, as described above. The fundamental group is $\pi_{1}(\tilde{M}) \cong N / N_{X}$.

Proof We may describe $\tilde{M}$ as a direct limit $\tilde{M}=\underline{\lim } \tilde{M}_{i}$, so we apply Corollary 2-7 and [15, Exercise 2.4.11] to deduce

$$
\pi_{1}(\tilde{M}) \cong \lim _{1} \pi_{1}\left(\tilde{M}_{i}\right) \cong \lim _{\longrightarrow} N / N_{X}=N / N_{X}
$$

We next show that $N / N_{X}$ is a subgroup of $\pi_{1}(M)$.

Corollary 2-9 Let $M$ be the toric origami manifold with orbit space $X$, let $\tilde{X}$ be the universal cover of $X$, and $\tilde{M}$ the toric origami manifold with boundary with orbit space $\tilde{X}$, as described above. Then there is an injection $N / N_{X} \hookrightarrow \pi_{1}(M)$.

Proof As noted above, we have a covering map $V: \tilde{M} \rightarrow M$ and therefore there is an injection $V_{*}: \pi_{1}(\tilde{M}) \hookrightarrow \pi_{1}(M)$. The result now follows from Corollary 2-8.

The group $N / N_{X}$ must be trivial or cyclic. When it is trivial, $\rho$ provides an isomorphism $\pi_{1}(M) \cong \pi_{1}(X)$ in Proposition 2-2. We now tackle the two separate cases when $N / N_{X}$ is finite and when it is isomorphic to $\mathbb{Z}$. 
Proposition 2-10 Let $M$ be the toric origami manifold with orbit space $X$. If $N / N_{X}$ is a finite cyclic group, then the surjection $\rho$ from Proposition 2-2 is an isomorphism.

Proof We know that $\pi_{1}(M) \cong\left(N / N_{X} \times \pi_{1}(X)\right) / \operatorname{ker}(\rho)$, and that the kernel $\operatorname{ker}(\rho) \subset$ $N / N_{X}$. Because $N / N_{X}$ is finite, we have an isomorphism $\pi_{1}(M) \cong \mathbb{Z} / k \mathbb{Z} \times F_{\ell}$, where $F_{\ell} \cong \pi_{1}(X)$ is a free group on $\ell$ generators. The image of $N / N_{X}$ under the injection $V_{*}$ must be in the $\mathbb{Z} / k \mathbb{Z}$ factor, since $F_{\ell}$ is free. The only way for the finite group $N / N_{X}$ to be a subgroup of $\mathbb{Z} / k \mathbb{Z} \cong\left(N / N_{X}\right) / \operatorname{ker}(\rho)$ is for $\operatorname{ker}(\rho)=\{\mathbb{1}\}$. This completes the proof.

Finally, we turn to the case where $N / N_{X} \cong \mathbb{Z}$. This situation turns out to be quite rigid.

Proposition 2-11 The quotient $N / N_{X} \cong \mathbb{Z}$ if and only if the toric origami manifold $M$ of dimension $2 n$ is equivariantly homeomorphic to $\mathbb{T}^{2} \times Y$, where $Y=Y_{F}$ is a toric symplectic manifold of dimension $2 n-2$, the torus $\mathbb{T}^{2}$ is a toric origami manifold.

Proof $(\Rightarrow)$ We begin by assuming that $N / N_{X} \cong \mathbb{Z}$. This means that $N_{\mathbb{R}} /\left(N_{X}\right)_{\mathbb{R}} \cong$ $\mathbb{R}$, and so $U=\left(N_{X}\right)_{\mathbb{R}}$ is a hyperplane in $N_{\mathbb{R}}=\mathbb{R}^{n}$. Let $u$ be a non-zero vector orthogonal to $U$.

We fix a choice of a single polytope $P=\Psi_{V}(v)$ in the moment image, and fix $F$ a fold facet of $P$, that is, $F=\Psi_{E}(e)$ for some edge $e$ in the template. Let $\eta$ denote the normal vector to $F$. Let $F_{1}, \ldots, F_{S}$ be the facets of $P$ adjacent to $F$, and let $\eta_{1}, \ldots, \eta_{s}$ denote the normal vectors to the facets. Because $P$ is a Delzant polytope, $\eta, \eta_{1}, \ldots, \eta_{s}$ span $N$. The vectors $\eta_{1}, \ldots, \eta_{s}$ must span a subspace of $U$. Combined with the fact that $P$ is Delzant, we may conclude that $\eta_{1}, \ldots, \eta_{s}$ span an $(n-1)-$ dimensional subspace, so they must span all of $U$. Each hyperplane $\eta_{i}^{\perp}$ contains $\mathbb{R} \cdot u$. Thus, the affine hyperplanes $\mathcal{H}_{i}$ that define the facets $F_{i}$ all contain an affine translation of $\mathbb{R} \cdot u$. This means that the intersection of affine half-spaces $\bigcap_{i=1}^{s} \mathcal{H}_{i}^{+}$ used to define part of $P$ can be described as an infinite prism

$$
\bigcap_{i=1}^{s} \mathcal{H}_{i}^{+}=F+\mathbb{R} \cdot u,
$$

where + denotes the Minkowski sum. There cannot be another non-fold facet of $P$ because the Delzant condition would force that facet to have a normal vector pointing out of $U$, contradicting the hypothesis that $N / N_{X} \cong \mathbb{Z}$. Therefore, there are only fold facets remaining, and because fold facets must be isolated, there can be only one additional fold facet $\widetilde{F}$ capping off $P$. Note that the infinite prism $\widetilde{F}+\mathbb{R} \cdot u$ is identical to $F+\mathbb{R} \cdot u$, and indeed $F$ and $\widetilde{F}$ have the same combinatorial type. This description 
as a subset of an infinite prism is valid for each polytope in the image of $\Psi_{V}$. Moreover, because adjacent polytopes must agree near their shared fold facet, the infinite prism is identical in each case. This implies that the moment image of $M$ is contained in the infinite prism $F+\mathbb{R} \cdot u$. Moreover the template graph must be a cycle.

For our fixed choice of $P$ and $F$, we have a hyperplane $U_{F}=\eta^{\perp} \cong \mathbb{R}^{n-1}$, with lattice $U_{F} \cap N$. Let $Y=Y_{F}$ denote the toric symplectic manifold of dimension $2 n-2$ corresponding to the Delzant polytope $F \subset U_{F}$. We now consider the closure $M_{j}$ in $M$ of a connected component $W_{j}$ of $M \backslash Z$. This corresponds to a vertex $v_{j}$ in the template graph, and hence a polytope $P_{j}=\Psi_{V}\left(v_{j}\right)$. We want to show that $M_{j}$ is equivariantly homeomorphic to $Y_{F} \times \mathbb{S}^{1} \times\left[a_{j}, b_{j}\right]$. To do so, we will think of constructing a toric symplectic manifold in the topological manner, by taking a quotient of $P \times \mathbb{T}$ by an equivalence relation to get $Y_{P}=P \times \mathbb{T} / \sim$. In this way, if $P_{j}=F \times\left[a_{j}, b_{j}\right]$, then we get a splitting of the symplectic cut piece $C_{j}=Y_{F} \times Y_{\left[a_{j}, b_{j}\right]}=Y_{F} \times \mathbb{S}_{\left[a_{j}, b_{j}\right]}^{2}$, and hence $M_{j}=Y_{F} \times \mathbb{S}^{1} \times\left[a_{j}, b_{j}\right]$.

For the general case, we proceed as follows. Let $h: F \times\left[a_{j}, b_{j}\right] \rightarrow P_{j}$ be a linear homeomorphism, preserving faces, and consider the homeomorphism $h \times \mathbb{1}_{\mathbb{T}^{n}}: F \times$ $\left[a_{j}, b_{j}\right] \times \mathbb{T}^{n} \rightarrow P_{j} \times \mathbb{T}^{n}$. The closure $M_{j}$ of $W_{j}$ is obtained from $P_{j} \times \mathbb{T}^{n}$ by collapsing the appropriate $\mathbb{S}^{1} \subset \mathbb{T}^{n}$ fibers over those facets of $P_{j}$ which are in the image $h\left(\partial F \times\left[a_{j}, b_{j}\right]\right)$. Note that the symplectic cut piece $C_{j}$ corresponding to $W_{j}$ would be obtained by further collapsing the appropriate $\mathbb{S}^{1} \subset \mathbb{T}^{n}$ fibers over the remaining facets of $P_{j}$, namely $h\left(F \times\left\{a_{j}\right\}\right)$ and $h\left(F \times\left\{b_{j}\right\}\right)$. In the topological construction, the circle subgroup of $\mathbb{T}^{n}$ that we collapse over a particular facet is indicated by the normal vector to that facet. Because the homeomorphism $h$ does not change the normal vectors to the facets in the image $h\left(\partial F \times\left[a_{j}, b_{j}\right]\right)$, the map $h \times \mathbb{1}_{\mathbb{T}^{n}}$ induces an equivariant homeomorphism between $Y_{F} \times \mathbb{S}^{1} \times\left[a_{j}, b_{j}\right]$ and $M_{j}$, as desired.

Thus, we have seen that the closure $M_{j}$ of each connected component of $M \backslash Z$ is a manifold with boundary homeomorphic to $Y_{F} \times \mathbb{S}^{1} \times\left[a_{j}, b_{j}\right]$, with two boundary components that correspond to the two fold facets of the polytope $P_{j}$. The whole manifold $M$ is obtained from the collection of $M_{j}$ by identifying them along their boundaries as prescribed by $\Psi_{E}$. Thus, we may deduce that $M$ is equivariantly homeomorphic to the union of the $Y_{F} \times \mathbb{S}^{1} \times\left[a_{j}, b_{j}\right]$ along their boundaries, and therefore is equivariantly homeomorphic to $Y_{F} \times \mathbb{T}^{2}$.

$(\Leftarrow)$ If $M$ is equivariantly homeomorphic to $\mathbb{T}^{2} \times Y$ with $Y$ toric symplectic and $\mathbb{T}^{2}$ toric origami, then its moment image is as described in the paragraphs above: see [5, Figure 14] for the moment image of a toric origami $\mathbb{T}^{2}$. Thus we must have that $N / N_{X} \cong \mathbb{Z}$. 
Definition 2-12 In the case where the quotient $N / N_{X} \cong \mathbb{Z}$ and $M \cong \mathbb{T}^{2} \times Y$, we call the toric origami manifold prismatic.

Corollary 2-13 If a toric origami manifold $M$ is prismatic, then its fundamental group is

$$
\pi_{1}(M)=\mathbb{Z}^{2}
$$

Proof The fundamental group is a homeomorphism invariant, and $\pi_{1}\left(\mathbb{T}^{2} \times Y\right)=$ $\pi_{1}\left(\mathbb{T}^{2}\right) \times \pi_{1}(Y)$, where $\pi_{1}\left(\mathbb{T}^{2}\right)=\mathbb{Z}^{2}$ and $Y$ is simply connected because it is a toric symplectic manifold.

We now have all the necessary ingredients to compute $\pi_{1}(M)$.

Theorem 2-14 Let $M$ be an orientable toric origami manifold with orbit space $X$, and let $N$ and $N_{X}$ be as in Definition 2-1. Then the fundamental group of $M$ is

$$
\pi_{1}(M) \cong N / N_{X} \times \pi_{1}(X) .
$$

Proof By Remark 2-3, Proposition 2-10 and Corollary 2-13, the only thing missing is to check that for $M$ prismatic, the fundamental group of the orbit space is $\pi_{1}(X)=\mathbb{Z}$. This is true because $X$ deformation retracts onto the template graph, which as remarked in the proof of Proposition 2-11 is a cycle.

In particular, this allows us to deduce that Masuda and Park's map $\rho$ [17] is an isomorphism.

Corollary 2-15 The epimorphism $\rho: N / N_{X} \times \pi_{1}(X) \rightarrow \pi_{1}(M)$ from Proposition 2-2 is an isomorphism.

Proof By Remark 2-3, Proposition 2-10, we are only left with checking that $\rho$ is an isomorphism when $M$ is prismatic. Recall that in that case $N / N_{X}=\mathbb{Z}$ and $\pi_{1}(X)=\mathbb{Z}$.

We know that $\pi_{1}(M) \cong\left(N / N_{X} \times \pi_{1}(X)\right) / \operatorname{ker}(\rho) \cong \mathbb{Z}^{2} / \operatorname{ker}(\rho)$, and that $\operatorname{ker}(\rho) \subset$ $N / N_{X} \cong \mathbb{Z}$. Since $\pi_{1}(M)=\mathbb{Z}^{2}$, the only possibility is that the kernel is the trivial subgroup $\operatorname{ker}(\rho)=\{\mathbb{1}\}$.

Another consequence of Theorem 2-14 is a characterization of simply connected toric origami manifolds. The following result indicates that the key assumption in our previous work [13], namely that the origami template be acyclic, is a natural topological hypothesis. 
Corollary 2-16 A toric origami manifold is simply connected if and only if its origami template is acyclic.

Proof If $M$ is a toric origami manifold and has at least one cycle in its template graph, then there must be at least one infinite cyclic factor in $\pi_{1}(M)$ and $M$ not simply connected.

If the template graph is acyclic, then the $\pi_{1}(X)$ factor of $\pi_{1}(M)$ is trivial. In addition, any polytope corresponding to a leaf of the template graph has at least one vertex not contained in a fold facet. By the Delzant condition at that vertex, the lattice quotient $N / N_{X}$ is trivial. Thus $\pi_{1}(M)=\{\mathbb{1}\}$ and $M$ is simply connected.

In Table 2.17 below, we show examples where $N / N_{X}$ takes on all possible types of group.

\begin{tabular}{|c|c|c|c|}
\hline$M$ & $\mathbb{S}^{2} \times \mathbb{S}^{1} \times \mathbb{S}^{1} \cong \mathbb{S}$ & $\mathbb{S}^{3} \times \mathbb{S}^{1}$ & $L(k ; 1) \times \mathbb{S}^{1}$ \\
\hline$\Phi(M)$ & & & \\
\hline$N / N_{X}$ & $\mathbb{Z}$ & $\{\mathbb{1}\}$ & $\mathbb{Z} / k \mathbb{Z}$ \\
\hline$\pi_{1}(M)$ & $\mathbb{Z} \times \mathbb{Z}$ & $\mathbb{Z}$ & $\mathbb{Z} / k \mathbb{Z} \times \mathbb{Z}$ \\
\hline
\end{tabular}

Table 2.17: Examples of the possible types of $N / N_{X}$. In each case, the template graph has two vertices, connected to one another by two edges. Each quotient space has two facets, with facet normals indicated in the figures.

Remark 2-18 The form of the fundamental group of a toric origami manifold given by Theorem 2-14 excludes certain manifolds from admitting such a structure. For example, a non-trivial finite cyclic group $\mathbb{Z} / k \mathbb{Z}$ cannot occur as the fundamental group of a toric origami manifold (as noted in [17, proof of Corollary 3.6] for the non-simply connected case). To verify this, we note that if $M$ is a toric origami manifold and has at least one cycle in its template graph, then there must be at least one infinite cyclic factor in $\pi_{1}(M)$. On the other hand, if the template graph is acyclic, then $M$ is simply connected by Corollary 2-16. Orlik and Raymond introduced manifolds so-called "of type $L$ " as some of the building blocks for 4-manifolds admitting toric actions [19]. More precisely, [20, Theorem VI.1] states that every orientable compact smooth 4-manifold that admits an effective smooth action of $\mathbb{T}^{2}$ with at least one fixed point is diffeomorphic to a connected sum of copies of $\mathbb{S}^{4}, \mathbb{C P}^{2}, \overline{\mathbb{C P}}^{2}, \mathbb{S}^{2} \times \mathbb{S}^{2}$, $\mathbb{S}^{1} \times \mathbb{S}^{3}, L_{n}$ and $L_{n}^{\prime}$ for $n \geq 2$. The fundamental group of the manifolds of type $L$ is $\pi_{1}\left(L_{n}\right)=\pi_{1}\left(L_{n}^{\prime}\right)=\mathbb{Z} / n \mathbb{Z}$ (see [20, page 296]), which implies that they do not admit a toric origami structure. It is easy to see that all the other building blocks do admit toric origami structures. 


\section{The cohomology of $M \backslash Z$}

In this section we obtain results about the cohomology of open toric symplectic manifolds of the form $Y \backslash \mathcal{B}$, where $Y$ is a compact toric symplectic manifold and $\mathcal{B}$ is a (not necessarily connected) codimension two toric symplectic submanifold of $Y$. This is exactly the form that the connected components of $M \backslash Z$ take. In Section 5, we will assemble these pieces in a Mayer-Vietoris sequence and deduce facts about the cohomology of $M$.

In this and the following section, we write

$$
\beta_{i}(X)=\operatorname{rank}\left(H_{i}(X ; \mathbb{Z})\right) \text { and } \beta^{i}(X)=\operatorname{rank}\left(H^{i}(X ; \mathbb{Z})\right)
$$

to denote the $i^{\text {th }}$ homology and cohomology Betti numbers of the space $X$, respectively. We begin by stating a result about the Euler characteristic of a manifold $Y \backslash \mathcal{B}$. This fact is known in greater generality; see for example [9, Section 4.5].

Proposition 3-1 The Euler characteristics of $Y$ and $\mathcal{B}$ are additive:

$$
\chi(Y \backslash \mathcal{B})=\chi(Y)-\chi(\mathcal{B})
$$

Proof We consider the long exact sequence for the pair $(Y, Y \backslash \mathcal{B})$, with integer coefficients understood:

$$
\cdots \rightarrow H_{*}(Y \backslash \mathcal{B}) \rightarrow H_{*}(Y) \rightarrow H_{*}(Y, Y \backslash \mathcal{B}) \rightarrow H_{*-1}(Y \backslash \mathcal{B}) \rightarrow \cdots
$$

By [12, Proposition 3.46], noting that $\mathcal{B}$ is compact and locally contractible and $Y$ is an orientable manifold, and by Poincaré duality for the manifold $\mathcal{B}$, we can replace the relative terms:

$$
H_{*}(Y, Y \backslash \mathcal{B}) \cong H^{2 n-*}(\mathcal{B}) \cong H_{*-2}(\mathcal{B}) .
$$

For simplicity of bookkeeping, we write $\beta_{-2}(\mathcal{B})=\beta_{-1}(\mathcal{B})=0$, since these ranks correspond, via the Poincaré duality in (3-3), to

$$
H_{-2}(\mathcal{B}) \cong H^{2 n}(\mathcal{B})=0 \text { and } H_{-1}(\mathcal{B}) \cong H^{2 n-1}(\mathcal{B})=0 .
$$

Taking the alternating sum of the ranks of the terms in the sequence (3-2), we obtain

$$
\begin{aligned}
0 & =\sum_{k=0}^{2 n}(-1)^{k}\left[\beta_{k}(Y \backslash \mathcal{B})-\beta_{k}(Y)+\beta_{k-2}(\mathcal{B})\right] \\
& =\sum_{k=0}^{2 n}(-1)^{k} \beta_{k}(Y \backslash \mathcal{B})-\sum_{k=0}^{2 n}(-1)^{k} \beta_{k}(Y)+\sum_{j=0}^{2 n-2}(-1)^{j} \beta_{j}(\mathcal{B}) \\
& =\chi(Y \backslash \mathcal{B})-\chi(Y)+\chi(\mathcal{B}) .
\end{aligned}
$$


We now prove a lemma related to [12, Proposition 3.46] that is a dual version of what is commonly called Alexander-Lefschetz duality. We adapt the very explicit proof given by Møller [18, Theorem 4.92] to this dual version, taking into account our special case that $\mathcal{B}$ is a submanifold of $Y$, suitably oriented.

Lemma 3-4 There is an isomorphism $H^{j}(Y, Y \backslash \mathcal{B} ; \mathbb{Z}) \cong H_{2 n-j}(\mathcal{B} ; \mathbb{Z})$.

Proof Let $U$ be an open neighborhood of $\mathcal{B}$ in $Y$. We begin by recalling the particulars of cap products. Both $Y$ and $\mathcal{B}$ are $\mathbb{Z}$-orientable manifolds, and so we have the following maps induced by taking a cap product with appropriate orientation classes.

(1) For the compact manifold $Y$, we have orientation class $\mu_{Y} \in H_{2 n}(Y ; \mathbb{Z})$, which gives

$$
H^{j}(Y ; \mathbb{Z}) \stackrel{\mu_{Y} \cap-}{\longrightarrow} H_{2 n-j}(Y ; \mathbb{Z}) .
$$

(2) For the manifold with boundary $Y \backslash U$, we have the relative orientation class $\mu_{Y \backslash U} \in H_{2 n}(Y \backslash \mathcal{B}, U \backslash \mathcal{B} ; \mathbb{Z})$, which gives

$$
H^{j}(Y \backslash \mathcal{B} ; \mathbb{Z}) \stackrel{\mu_{Y \backslash U} \cap-}{\longrightarrow} H_{2 n-j}(Y \backslash \mathcal{B}, U \backslash \mathcal{B} ; \mathbb{Z}) .
$$

(3) For the compact manifold $\mathcal{B}$, we have the relative orientation class $\mu_{\mathcal{B}} \in$ $H_{2 n}(U, U \backslash \mathcal{B} ; \mathbb{Z})$. By pre-composing with the excision isomorphism, we have

$$
H^{j}(Y, Y \backslash \mathcal{B} ; \mathbb{Z}) \cong H^{j}(U, U \backslash \mathcal{B} ; \mathbb{Z}) \stackrel{\mu_{\mathcal{B}} \cap-}{\longrightarrow} H_{2 n-j}(U ; \mathbb{Z})
$$

We use these maps to produce a diagram, with integer coefficients,

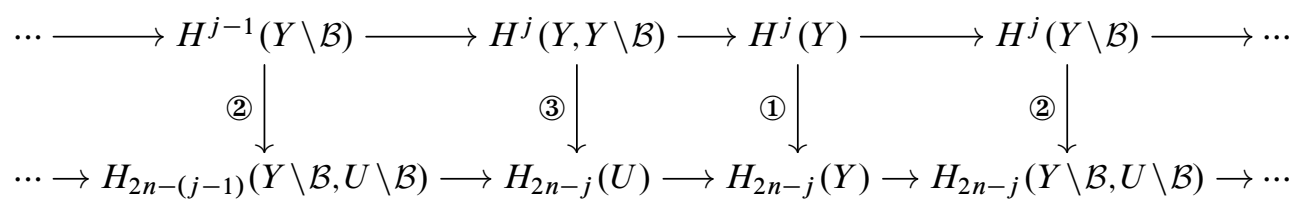

where the top row is the long exact sequence of the pair $(Y, Y \backslash \mathcal{B})$, and the bottom row is the long exact sequence of the pair $(Y, U)$ where the terms $H_{k}(Y, U)$ are replaced by $H_{k}(Y \backslash \mathcal{B}, U \backslash \mathcal{B})$ via excision. This diagram commutes: the "up to sign" discrepancy in [18, proof of Theorem 4.92] disappears because we may choose $Y$ and $\mathcal{B}$ to be compatibly oriented. 
We next take a limit over the poset $\mathcal{U}$ of neighborhoods $U$ containing $\mathcal{B}$ to obtain a limit diagram

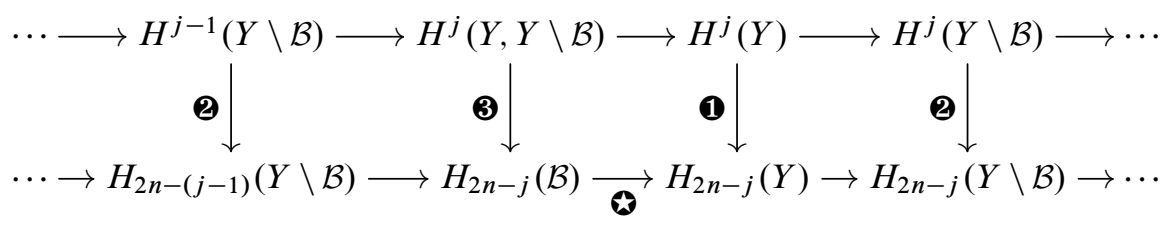

which still commutes, and the bottom sequence remains exact under the limit. We observe that the map is induced by inclusion. We also note that $(1)$ and 2 are Poincare duality isomorphisms. We now apply the five lemma to deduce that $\mathbf{3}$ is an isomorphism, completing the proof.

We return to the long exact sequence of the pair $(Y, Y \backslash \mathcal{B})$, which is the top row in the diagram (3-5), with integer coefficients understood. The toric symplectic manifold $Y$ has cohomology concentrated in even degrees, up to degree $2 n$. The space $\mathcal{B}$ is a disjoint union of toric symplectic manifolds, therefore its homology is concentrated in even degrees up to degree $2 n-2$. Then by Lemma 3-4 the long exact sequence splits into 4-term exact sequences, with integer coefficients,

$$
0 \rightarrow H^{2 k-1}(Y \backslash \mathcal{B}) \rightarrow H^{2 k}(Y, Y \backslash \mathcal{B}) \stackrel{\varphi_{k}}{\longrightarrow} H^{2 k}(Y) \rightarrow H^{2 k}(Y \backslash \mathcal{B}) \rightarrow 0 .
$$

Thus, we may always identify $H^{2 k-1}(Y \backslash \mathcal{B}) \cong \operatorname{ker}\left(\varphi_{k}\right)$ and $H^{2 k}(Y \backslash \mathcal{B}) \cong \operatorname{coker}\left(\varphi_{k}\right)$.

Let us now look more carefully at the map $\varphi_{k}$. We have a diagram

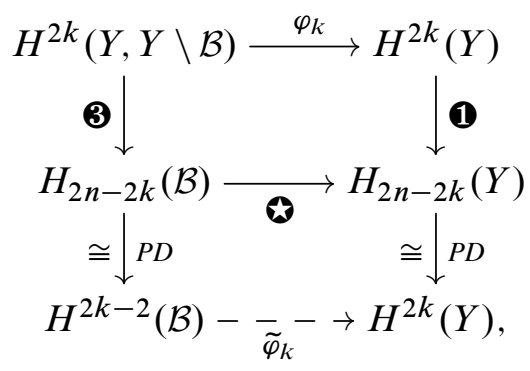

where all vertical maps are isomorphisms, and we define $\tilde{\varphi}_{k}$ to be the map that makes the bottom square commute. Recall from the comments after (3-5) that is the natural map induced by the inclusion $i: \mathcal{B} \hookrightarrow Y$. The homology groups of $Y$ and $\mathcal{B}$ are isomorphic to the Chow homology groups of those varieties. The Chow groups of smooth toric varieties are very explicitly understood: they are spanned by classes, one for each $\mathbb{T}$-invariant subvariety. A subvariety in $\mathcal{B}$ may be regarded as a subvariety of $Y$, and so the map maps the corresponding class on $\mathcal{B}$ to the class on $Y$. 
When we apply Poincaré duality, we have very explicit presentations of the cohomology rings $H^{*}(Y ; \mathbb{Z})$ and $H^{*}(\mathcal{B} ; \mathbb{Z})$ as the face rings of the corresponding polytopes, modulo linear relations. That is, when the moment polytope $\Delta_{Y}$ for $Y$ has facets $F_{1}, \ldots, F_{d}$, we may describe

$$
H^{*}(Y ; \mathbb{Z}) \cong \frac{\mathbb{Z}\left[y_{1}, \ldots, y_{d}\right]}{\left.\left\langle\prod_{i \in I} y_{i}\right| \bigcap_{i \in I} F_{i}=\varnothing+\text { linear terms }\right\rangle},
$$

where each $y_{i}$ has degree 2, and is the Poincare dual of the codimension 2 toric symplectic submanifold corresponding to the facet $F_{i}$. The linear terms are determined by the geometry of the normal vectors to the facets. We note, for bookkeeping purposes, that there are precisely $n=\frac{1}{2} \operatorname{dim}(Y)$ independent linear relations. That is, $\operatorname{rank}\left(H^{2}(Y)\right)=d-n$. Equation (3-8) is the content of the Danilov-Jurkiewicz theorem, which is carefully described in [6, Theorem 12.4.4].

For a connected component $B_{S} \subset \mathcal{B}$, the moment image $\Delta_{B_{S}}$ of $B_{S}$ is one of the facets $F_{s}$. The facets of $\Delta_{B_{s}}$ are each an intersection $F_{S} \cap F_{j}$, and so as above, we may describe

$$
H^{*}\left(B_{s} ; \mathbb{Z}\right) \cong \frac{\mathbb{Z}\left[b_{j_{1}}, \ldots, b_{j_{m}}\right]}{\left.\left\langle\prod_{i \in I} b_{j_{i}}\right| \bigcap_{i \in I}\left(F_{j_{i}} \cap F_{S}\right)=\varnothing+\text { linear terms }\right\rangle} .
$$

Because the $y_{i}$ and $b_{i}$ are Poincaré duals to explicit submanifolds of $Y$ and $B_{S}$ respectively, and because is induced by inclusion, we may derive an explicit formula for $\tilde{\varphi}_{k}$. For the component $B_{s} \subset \mathcal{B}$ and a single monomial $\prod_{i \in I} b_{j_{i}} \in H^{2 k-2}\left(B_{s} ; \mathbb{Z}\right)$,

$$
\tilde{\varphi}_{k}\left(\prod_{i \in I} b_{j_{i}}\right)=y_{s} \cdot \prod_{i \in I} y_{j_{i}}
$$

This is not a ring map, as expected.

The following definition extends the notion in Definition 2-12 of prismatic origami manifolds to a wider context. Let $A$ be an open toric symplectic manifold with open moment polytope $\Delta_{A}$. The lattice $N_{\Delta_{A}}$ is the sublattice of $N$ spanned by the normal vectors to the facets of $\Delta_{A}$.

Definition 3-9 An open toric symplectic manifold $A$ with moment polytope $\Delta_{A}$ is prismatic if the quotient of lattices $N / N_{\Delta_{A}}$ is $\mathbb{Z}$.

We now turn to $\widetilde{\varphi}_{1}: H^{0}(\mathcal{B} ; \mathbb{Z}) \rightarrow H^{2}(Y ; \mathbb{Z})$. The group $H^{0}(\mathcal{B} ; \mathbb{Z}) \cong \mathbb{Z}^{r}$ has one generator for each connected component of $\mathcal{B}$, each corresponding to a facet in $\Delta_{Y}$. The group $H^{2}(Y ; \mathbb{Z})$ has one generator for each facet of $\Delta_{Y}$, modulo linear relations. By our explicit description above, the map $\tilde{\varphi}_{1}$ takes the generator of $H^{0}(\mathcal{B})$ corresponding 
to a facet $F_{S}$ of the polytope $\Delta_{Y}$ to the generator $y_{S} \in H^{2}(Y ; \mathbb{Z})$ corresponding to the same facet. We may use our explicit description of $\widetilde{\varphi}_{1}$ to determine $\operatorname{ker}\left(\widetilde{\varphi}_{1}\right) \cong$ $H^{1}(Y \backslash \mathcal{B} ; \mathbb{Z})$ in general.

Lemma 3-10 The kernel of the map $\tilde{\varphi}_{1}: H^{0}(\mathcal{B} ; \mathbb{Z}) \rightarrow H^{2}(Y ; \mathbb{Z})$ is $\operatorname{ker}\left(\widetilde{\varphi}_{1}\right) \cong \mathbb{Z}$ if $Y \backslash \mathcal{B}$ is prismatic and trivial otherwise.

Proof Without loss of generality, we may assume that $\mathcal{B}$ corresponds to the disjoint union of facets $F_{1}, \ldots, F_{r}$ of $\Delta_{Y}$. Let $u_{1}, \ldots, u_{d}$ be the primitive outward pointing normals to all the facets of $\Delta_{Y}$. Then

$$
H^{0}(\mathcal{B} ; \mathbb{Z})=\mathbb{Z} x_{1} \oplus \cdots \oplus \mathbb{Z} x_{r} \quad \text { and } \quad H^{2}(Y ; \mathbb{Z})=\mathbb{Z} y_{1} \oplus \cdots \oplus \mathbb{Z} y_{d} / J,
$$

where $J$ is the ideal of linear relations, which are $\sum_{i=1}^{d}\left\langle v, u_{i}\right\rangle y_{i}$, for all $v \in N$. Henceforth, we will abuse notation, and let $y_{i}$ denote the equivalence class $y_{i}+J \in$ $H^{2}(Y ; \mathbb{Z})$.

The map $\tilde{\varphi}_{1}$ is given by $\tilde{\varphi}_{1}\left(x_{i}\right)=y_{i}$. Therefore an element $\sum_{i=1}^{r} a_{i} x_{i} \in H^{0}(\mathcal{B} ; \mathbb{Z})$ is in the kernel of $\tilde{\varphi}_{1}$ if and only if there exists a $v \in N$ such that

$$
\sum_{i=1}^{r} a_{i} y_{i}=\sum_{i=1}^{d}\left\langle v, u_{i}\right\rangle y_{i} .
$$

Equivalently, $v \in N$ must satisfy

$$
\begin{cases}\left\langle v, u_{i}\right\rangle=a_{i} & \text { for } i=1, \ldots, r, \\ \left\langle v, u_{i}\right\rangle=0 & \text { for } i=r+1, \ldots, d .\end{cases}
$$

The second half of (3-11) means that $v \in\left(N_{\Delta_{Y \backslash \mathcal{B}}}\right)^{\perp}$.

If $Y \backslash \mathcal{B}$ is not prismatic, then the lattice quotient $N / N_{\Delta_{Y \backslash \mathcal{B}}}$ is either trivial or finite cyclic, which implies that $\left(N_{\Delta_{Y \backslash \mathcal{B}}}\right)^{\perp}$ is trivial and $\operatorname{so} \operatorname{ker}\left(\widetilde{\varphi}_{1}\right)$ is trivial as well.

If $Y \backslash \mathcal{B}$ is prismatic, then $\left(N_{\Delta_{Y \backslash \mathcal{B}}}\right)^{\perp} \cong \mathbb{Z}$ and a generator $v$ of $\left(N_{\Delta_{Y \backslash \mathcal{B}}}\right)^{\perp}$ will provide a non-zero element $\sum_{i=1}^{d}\left\langle v, u_{i}\right\rangle y_{i} \in J$, and therefore gives a generator $\sum_{i=1}^{r}\left\langle v, u_{i}\right\rangle x_{i}$ of $\operatorname{ker}\left(\widetilde{\varphi}_{1}\right)$.

We can now prove the main result of the section.

Theorem 3-12 Let $Y$ be a $2 n$-dimensional toric symplectic manifold with moment polytope $\Delta_{Y}$, and let $d$ denote the number of facets of $\Delta_{Y}$. Let $\mathcal{B}$ be a non-empty codimension 2 toric symplectic submanifold with $r$ connected components. We may compute the following Betti numbers of $Y \backslash \mathcal{B}$. 


\begin{tabular}{r|cc} 
& $Y \backslash \mathcal{B}$ prismatic & $Y \backslash \mathcal{B}$ not prismatic \\
\hline$\beta^{0}(Y \backslash \mathcal{B})$ & 1 & 1 \\
$\beta^{1}(Y \backslash \mathcal{B})$ & 1 & 0 \\
$\beta^{2}(Y \backslash \mathcal{B})$ & $d-n-1$ & $d-n-r$ \\
$\beta^{2 n-1}(Y \backslash \mathcal{B})$ & 1 & $r-1$ \\
$\beta^{2 n}(Y \backslash \mathcal{B})$ & 0 & 0
\end{tabular}

Proof We begin by noting that if $Y \backslash \mathcal{B}$ is prismatic then $\mathcal{B}$ necessarily has $r=2$ connected components. Using this, we will determine all rows simultaneously in the prismatic and non-prismatic cases.

(0) $\beta^{0}(Y \backslash \mathcal{B})$ The manifold $Y \backslash B$ is connected, so $\beta^{0}(Y \backslash \mathcal{B})=1$.

(1) $\beta^{1}(Y \backslash \mathcal{B})$ Recall that $\operatorname{ker}\left(\widetilde{\varphi}_{1}\right) \cong H^{1}(Y \backslash \mathcal{B}$; $\mathbb{Z})$. Lemma 3-10 says $\beta^{1}(Y \backslash \mathcal{B})=1$ when $Y \backslash \mathcal{B}$ is prismatic, and $\beta^{1}(Y \backslash \mathcal{B})=0$ otherwise.

(2) $\beta^{2}(Y \backslash \mathcal{B})$ We now identify the terms of (3-6) in the case $k=1$ :

$$
\begin{aligned}
0 \rightarrow H^{1}(Y \backslash \mathcal{B}) \rightarrow H^{2}(Y, Y \backslash \mathcal{B}) & \\
& \cong H^{2}(\mathcal{B}) \cong \mathbb{Z}^{r} \rightarrow H^{2}(Y) \cong \mathbb{Z}^{d-n} \rightarrow H^{2}(Y \backslash \mathcal{B}) \rightarrow 0 .
\end{aligned}
$$

A dimension count proves that

$$
\beta^{2}(Y \backslash \mathcal{B})=\beta^{1}(Y \backslash \mathcal{B})+d-n-r .
$$

Substituting $\beta^{1}(Y \backslash \mathcal{B})$, and $r=2$ in the prismatic case, completes the calculation.

(3) $\beta^{2 n}(Y \backslash \mathcal{B})$ We note that $Y \backslash \mathcal{B}$ is homotopy equivalent to a manifold $X$ with boundary $\mathcal{Z}$. Poincaré duality for manifolds with boundary implies that

$$
H^{2 n}(Y \backslash \mathcal{B} ; \mathbb{Z}) \cong H^{2 n}(X ; \mathbb{Z}) \cong H_{0}(X, \mathcal{Z} ; \mathbb{Z})
$$

We note that relative cohomology $H_{0}(X, \mathcal{Z} ; \mathbb{Z}) \cong \widetilde{H}_{0}((X / \mathcal{Z} ; \mathbb{Z})$, and this is 0 because $X / \mathcal{Z}$ is connected.

(4) $\beta^{2 n-1}(Y \backslash \mathcal{B})$ We now identify the terms of (3-6) in the case $k=n$ :

$$
\begin{aligned}
0 \rightarrow H^{2 n-1}(Y \backslash \mathcal{B}) \rightarrow H^{2 n}(Y, Y \backslash \mathcal{B}) & \\
& \cong H^{2 n-2}(\mathcal{B}) \cong \mathbb{Z}^{r} \rightarrow H^{2 n}(Y) \cong \mathbb{Z} \rightarrow H^{2 n}(Y \backslash \mathcal{B})=0 \rightarrow 0 .
\end{aligned}
$$

We have the rightmost equality $H^{2 n}(Y \backslash \mathcal{B})=0$ by the previous computation. A dimension count now proves that $\beta^{2 n-1}(Y \backslash \mathcal{B})=r-1$. Substituting $r=2$ in the prismatic case completes the calculation. 
We note that when $n=2$, Theorem 3-12 gives all the Betti numbers of $Y \backslash \mathcal{B}$. Even in higher dimensions, in specific examples, it is often tractable to compute the various maps $\widetilde{\varphi}_{k}$, and to compute all of the Betti numbers, as well as torsion in the cohomology groups $H^{*}(Y \backslash \mathcal{B} ; \mathbb{Z})$. We conclude the section with such an example.

Example 3-13 Let $Y$ be the toric variety $\mathbb{C P}^{1} \times \mathbb{C P}^{1} \times \mathbb{C P}^{1}$ blown up at one fixed point. This has the moment polytope shown in Figure 3.14.

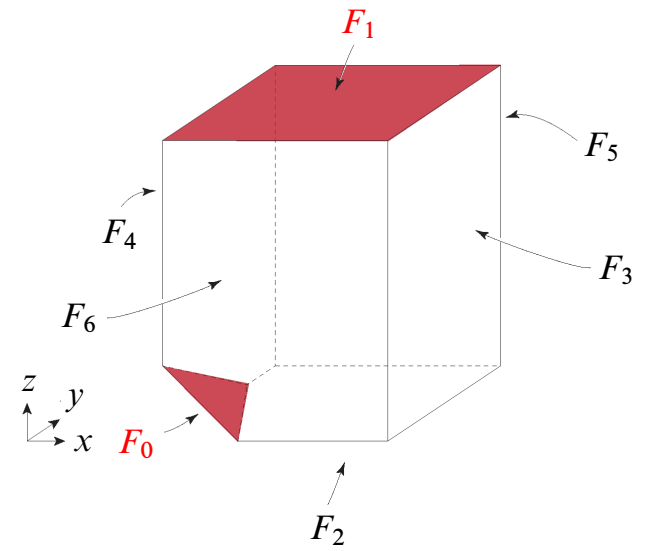

Figure 3.14: The moment map image for the $\mathbb{T}^{3}$ action on $\mathbb{C P}^{1} \times \mathbb{C P}^{1} \times \mathbb{C} P^{1}$ blown up at one fixed point. The polytope is a cube truncated at one vertex.

In our calculations below, we will use the linear relations to simplify the presentations of the cohomology rings; that is, we will use them to reduce the number of degree 2 generators. We have

$$
\begin{aligned}
H^{*}(Y ; \mathbb{Z}) & =\frac{\mathbb{Z}\left[y_{0}, y_{1}, y_{2}, y_{3}, y_{4}, y_{5}, y_{6}\right]}{\left\langle\begin{array}{l}
y_{1} y_{2}, y_{3} y_{4}, y_{5} y_{6}, y_{0} y_{1}, y_{0} y_{3}, y_{0} y_{5}, y_{2} y_{4} y_{6}, \\
-y_{0}+y_{3}-y_{4},-y_{0}+y_{5}-y_{6},-y_{0}+y_{1}-y_{2}
\end{array}\right\rangle} . \\
& \cong \frac{\mathbb{Z}\left[y_{0}, y_{2}, y_{4}, y_{6}\right]}{\left\langle\begin{array}{c}
y_{0}^{2}-y_{2}^{2}, y_{0}^{2}-y_{4}^{2}, y_{0}^{2}-y_{6}^{2}, \\
y_{0}^{2}+y_{0} y_{2}, y_{0}^{2}+y_{0} y_{4}, y_{0}^{2}+y_{0} y_{6}, y_{2} y_{4} y_{6}
\end{array}\right\rangle} .
\end{aligned}
$$

We next compute the cohomology of $\mathcal{B}$ :

$$
\begin{aligned}
& H^{*}\left(B_{0} ; \mathbb{Z}\right)=\frac{\mathbb{Z}\left[b_{2}, b_{4}, b_{6}\right]}{\left\langle b_{2} b_{4} b_{6}, b_{4}-b_{6}, b_{2}-b_{4}\right\rangle} \cong \frac{\mathbb{Z}\left[b_{2}\right]}{\left\langle b_{2}^{3}\right\rangle}, \\
& H^{*}\left(B_{1} ; \mathbb{Z}\right)=\frac{\mathbb{Z}\left[b_{3}, b_{4}, b_{5}, b_{6}\right]}{\left\langle b_{3} b_{4}, b_{5} b_{6}, b_{3}-b_{4}, b_{5}-b_{6}\right\rangle} \cong \frac{\mathbb{Z}\left[b_{4}, b_{6}\right]}{\left\langle b_{4}^{2}, b_{6}^{2}\right\rangle} .
\end{aligned}
$$


Putting these two calculations together, and using $x_{0}$ and $x_{1}$ as degree-zero dummy variable placeholders, we have

$$
H^{*}(\mathcal{B} ; \mathbb{Z})=x_{0} \frac{\mathbb{Z}\left[b_{2}\right]}{\left\langle b_{2}^{3}\right\rangle} \oplus x_{1} \frac{\mathbb{Z}\left[b_{4}, b_{6}\right]}{\left\langle b_{4}^{2}, b_{6}^{2}\right\rangle} .
$$

We know that $\tilde{\varphi}_{k}$ maps $b_{i}$ to $y_{i}$, and $x_{i}$ to $y_{i}$. It is only a matter of bookkeeping to compute

$$
\begin{aligned}
& H^{0}(Y \backslash \mathcal{B} ; \mathbb{Z})=\mathbb{Z}, \\
& H^{1}(Y \backslash \mathcal{B} ; \mathbb{Z})=\operatorname{ker}\left(\widetilde{\varphi}_{1}\right)=0, \\
& H^{2}(Y \backslash \mathcal{B} ; \mathbb{Z})=\operatorname{coker}\left(\widetilde{\varphi}_{1}\right)=\operatorname{span}\left\{y_{4}, y_{6}\right\} \cong \mathbb{Z}^{2}, \\
& H^{3}(Y \backslash \mathcal{B} ; \mathbb{Z})=\operatorname{ker}\left(\widetilde{\varphi}_{2}\right)=0, \\
& H^{4}(Y \backslash \mathcal{B} ; \mathbb{Z})=\operatorname{coker}\left(\widetilde{\varphi}_{2}\right)=\operatorname{span}\left\{y_{4} y_{6}\right\} \cong \mathbb{Z}, \\
& H^{5}(Y \backslash \mathcal{B} ; \mathbb{Z})=\operatorname{ker}\left(\widetilde{\varphi}_{3}\right)=\operatorname{span}\left\{x_{0} b_{2}^{2}-x_{1} b_{4} b_{6}\right\} \cong \mathbb{Z}, \\
& H^{6}(Y \backslash \mathcal{B} ; \mathbb{Z})=\operatorname{coker}\left(\widetilde{\varphi}_{3}\right)=0 .
\end{aligned}
$$

\section{The cohomology of $Z$}

In this section we describe a method for computing the cohomology groups of connected components of the fold $Z$. It suffices to make computations one connected component $\mathcal{Z}$ at a time. Let $\mathbb{T} \subset Y$ denote a toric symplectic manifold and $\mathcal{B}$ a $\mathbb{T}$-invariant connected codimension two symplectic submanifold of $Y$. The unit sphere bundle $v_{\mathbb{1}}(\mathcal{B} \subset Y)$ of the normal bundle $v(\mathcal{B} \subset Y)$ is equivariantly homeomorphic to the circle bundle $\mathbb{S}^{1} \hookrightarrow \mathcal{Z} \rightarrow \mathcal{B}$. We will use the ordinary and equivariant Gysin sequences for this bundle to describe an explicit way to determine the Betti numbers of $\mathcal{Z}$. In Section 5, we will combine this with results of Section 3 to compute the Betti numbers of a toric origami manifold in some cases.

The action $\mathbb{T} \subset Y$ is toric with moment polytope $\Delta_{Y} \subset \mathbb{R}^{n}$, and the submanifold $\mathcal{B}$ is fixed by a circle $\mathbb{S}^{1} \subset \mathbb{T}$. The residual action $\mathbb{T}_{\mathcal{B}}=\mathbb{T} / \mathbb{S}^{1} \subset \mathcal{B}$ is a toric action with moment polytope $\Delta_{\mathcal{B}}$. Order the facets $F_{1}, \ldots, F_{d}$ so that $F_{m+1}$ is the moment image of $B$, and $F_{1}, \ldots, F_{m}$ are the facets of $\Delta_{Y}$ with non-empty intersection with $F_{m+1}$. Thus, the facets of $\Delta_{\mathcal{B}}$ are precisely $F_{1} \cap F_{m+1}, \ldots, F_{m} \cap F_{m+1}$. We will study the $\mathbb{T}$-equivariant cohomology rings of $\mathcal{Z}$ and $\mathcal{B}$. Because $\mathbb{S}^{1} \mathrm{C} \mathcal{Z}$ is free, we have ring isomorphisms

$$
H_{\mathbb{T}}^{*}(\mathcal{Z}) \cong H_{\mathbb{T} / \mathbb{S}^{1}}^{*}\left(\mathcal{Z} / \mathbb{S}^{1}\right) \cong H_{\mathbb{T}_{\mathcal{B}}}^{*}(\mathcal{B}),
$$

so we will also need an explicit description of $H_{\mathbb{T}_{\mathcal{B}}}^{*}(\mathcal{B})$. 
The equivariant cohomology of a toric variety is given by the face ring of its moment polytope, so we have two face ring presentations

$$
\begin{aligned}
& H_{\mathbb{T}}^{*}(Y ; \mathbb{Z}) \cong \frac{\mathbb{Z}\left[y_{1}, \ldots, y_{d}\right]}{\left\langle\prod_{i \in I} y_{i} \mid \bigcap_{i \in I} F_{i}=\varnothing\right\rangle}, \\
& H_{\mathbb{T}_{\mathcal{B}}}^{*}(\mathcal{B} ; \mathbb{Z}) \cong \frac{\mathbb{Z}\left[b_{1}, \ldots, b_{m}\right]}{\left\langle\prod_{i \in I} b_{i} \mid \bigcap_{i \in I}\left(F_{i} \cap F_{m+1}\right)=\varnothing\right\rangle},
\end{aligned}
$$

where $y_{i}$ and $b_{i}$ are the degree-two generators Poincaré dual to the codimension two symplectic submanifolds corresponding to the facets of $\Delta_{Y}$ and $\Delta_{\mathcal{B}}$ respectively. We are abusing notation slightly here. The indexing set $I$ on the left is a subset of $\{1, \ldots, d\}$ and on the right a subset of $\{1, \ldots, m\}$.

The action $\mathbb{T} \subset \mathcal{B}$ is not effective, since $\mathbb{S}^{1}$ fixes $\mathcal{B}$ (pointwise). As vector spaces,

$$
H_{\mathbb{T}}^{*}(\mathcal{B}) \cong H_{\mathbb{B}_{\mathcal{B}}}^{*}(\mathcal{B}) \otimes H_{\mathbb{S}^{1}}^{*}(\mathrm{pt}),
$$

over any coefficient ring. To find a ring presentation, we consider the restriction map

$$
H_{\mathbb{T}}^{*}(Y ; \mathbb{Z}) \rightarrow H_{\mathbb{T}}^{*}(\mathcal{B} ; \mathbb{Z})
$$

This map is easily seen to be surjective, for example by an equivariant version of [13, Lemma 3.4]. The classes $y_{i}$ for $i \geq m+2$ are in the kernel of this restriction map; this can be seen by recognizing that the restriction of $y_{i}$ to the fixed points restricts to 0 at the fixed points in $\mathcal{B}$. The remaining generators $y_{1}, \ldots, y_{m+1}$ are non-zero in $H_{\mathbb{T}}^{*}(\mathcal{B} ; \mathbb{Z})$, and are multiplicative generators. Abusing notation slightly, we call the images of those classes $b_{1}, \ldots, b_{m+1}$ respectively. Keeping track of which relations contribute, we find

$$
H_{\mathbb{T}}^{*}(\mathcal{B} ; \mathbb{Z}) \cong \frac{\mathbb{Z}\left[b_{1}, \ldots, b_{m+1}\right]}{\left\langle\prod_{i \in I} b_{i} \mid \bigcap_{i \in I}\left(F_{i} \cap F_{m+1}\right)=\varnothing\right\rangle} .
$$

With this presentation, the restriction map $H_{\mathbb{T}}^{*}(Y ; \mathbb{Z}) \rightarrow H_{\mathbb{T}}^{*}(\mathcal{B} ; \mathbb{Z})$ sends $y_{i}$ to $b_{i}$ for values of $i=1, \ldots, m+1$, and sends $y_{i}$ to 0 for $i>m+1$. We may think of $b_{m+1}$ as the generator of $H_{\mathbb{S}^{1}}^{*}(p t)$ in the decomposition (4-2); it is not involved in any of the residual multiplicative relations.

The natural map

$$
H_{\mathbb{T}}^{*}(\mathcal{B} ; \mathbb{Z}) \rightarrow H_{\mathbb{T}_{\mathcal{B}}}^{*}(\mathcal{B} ; \mathbb{Z})
$$

has kernel

$$
\left\langle\sum_{i=1}^{m+1}\left\langle u_{i}, u_{m+1}\right\rangle b_{i}\right\rangle
$$

where $u_{i}$ is the outward pointing primitive normal to $F_{i}$ in $\mathbb{R}^{n}$. 
We now turn to the ordinary and equivariant Gysin sequences for the circle bundle

$$
\mathbb{S}^{1} \hookrightarrow \mathcal{Z} \stackrel{\pi}{\longrightarrow} \mathcal{B} \text {. }
$$

Both the ordinary and equivariant cohomology of $\mathcal{B}$ are concentrated in even degrees, so both sequences split into four-term sequences. They fit into the following commutative diagram with coefficients in $\mathbb{Z}$ :

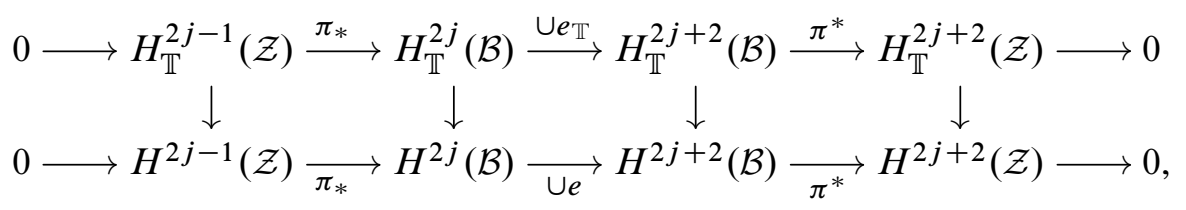

where $e_{\mathbb{T}}$ denotes the equivariant Euler class of the Gysin sequence, $e$ denotes the ordinary Euler class, $\pi_{*}$ is the push-forward map of $\pi: \mathcal{Z} \rightarrow \mathcal{B}$, and $\pi^{*}$ is the pullback of $\pi$. We note that the term $H_{\mathbb{T}}^{2 j-1}(\mathcal{Z})=0$, because the right-hand side of (4-1) is concentrated in even degrees. Thus we have

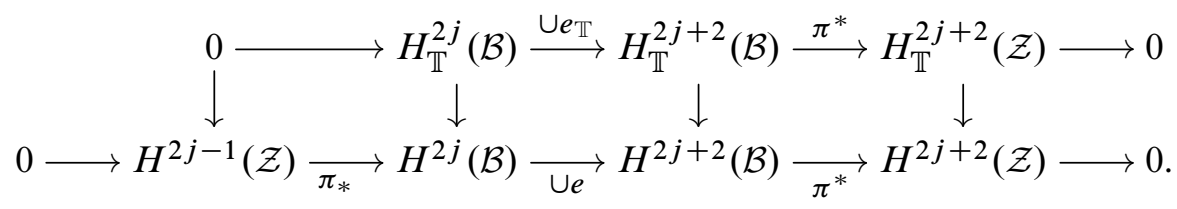

We note that the circle bundle $\pi: \mathcal{Z} \rightarrow \mathcal{B}$ is equivariantly homeomorphic to the unit sphere bundle $v_{\mathbb{1}}(\mathcal{B} \subset Y)$ in the normal bundle $v(\mathcal{B} \subset Y)$, so the Euler class and equivariant Euler class of the circle bundle are the same as the first Chern class and equivariant first Chern class of $v(\mathcal{B} \subset Y)$. Thus, in the presentation (4-3), the equivariant Euler class of the bundle $\mathcal{Z} \rightarrow \mathcal{B}$ is precisely the class $e_{\mathbb{T}}=b_{m+1}$. In the top row of (4-5), we see that $e_{\mathbb{T}}=b_{m+1}$ is a non-zero divisor. As noted above, we may think of $b_{m+1}$ as the generator of $H_{\mathbb{S}^{1}}^{*}(\mathrm{pt})$ in the decomposition (4-2).

We now turn to the ordinary Euler class. By naturality of characteristic classes, the ordinary Euler class $e$ is the image of $b_{m+1}$ in $H^{*}(\mathcal{B})$. In terms of the generators $b_{1}, \ldots, b_{m}$ of $H^{*}(\mathcal{B})$, by (4-4) we have

$$
b_{m+1}=-\sum_{i=1}^{m} \frac{\left\langle u_{i}, u_{m+1}\right\rangle}{\left\langle u_{m+1}, u_{m+1}\right\rangle} b_{i} .
$$

The right-hand side of the above expression appears to have rational coefficients; because the Euler class is an integral class, we may deduce that it must have integer coefficients.

We conclude this section with a few remarks about how these calculations may be used to understand $H^{*}(\mathcal{Z})$. 
Remark 4-6 We have explicit presentations for $H_{\mathbb{T}}^{*}(\mathcal{B})$ and $H^{*}(\mathcal{B})$, as well as all of the maps in (4-5). Thus, in order to determine the Betti numbers of $\mathcal{Z}$ in any particular example, one can pose the question, "what are the ranks of $\operatorname{ker}(\cup e), \operatorname{coker}(\cup e)$ and coker $\left(\cup e_{\mathbb{T}}\right)$ ?" to a program like Macaulay2.

Remark 4-7 In some special cases, it may be possible to determine the ring structure for $H^{*}(\mathcal{Z})$. For example, if $\mathcal{Z}$ is a good contact toric manifold, or more generally, if $b_{m+1} \in H^{2}(\mathcal{B})$ is a Lefschetz class, one can apply the results of Luo [14] to determine the cohomology ring $H^{*}(\mathcal{Z})$. We do not know if there is a general combinatorial criterion in terms of the polytope which guarantees that $b_{m+1}$ is a Lefschetz class. The manifold $\mathcal{Z}$ is a good contact toric manifold when $\operatorname{dim}(\mathcal{Z}) \geq 5$, and if the facets adjacent to $\Delta_{\mathcal{B}}$ form a cone so that we can obtain $\Delta_{\mathcal{B}}$ by chopping off a vertex of that cone. An example and non-example are shown in Figure 4.8.
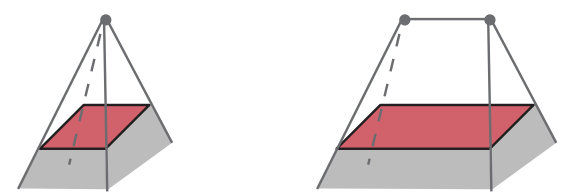

Figure 4.8: Left: a facet for which $\mathcal{Z}$ is good contact toric. The adjacent facets extend to a cone whose vertex we can chop to obtain $\Delta_{\mathcal{B}}$. Right: a facet for which $\mathcal{Z}$ is not good contact toric (with the specified fold form $\omega$ ). The adjacent facets do not form a cone.

Remark 4-9 The manifold $\mathcal{Z}$ is an almost contact manifold. Indeed, let $i: \mathcal{Z} \hookrightarrow M$ be the inclusion. If we let $v$ denote a nowhere vanishing section of $\mathcal{Z} \rightarrow \mathcal{B}$, and $\alpha \in \Omega^{1}(\mathcal{Z})$ a 1 -form defined by $\iota_{v} \alpha=1$, then $\alpha \wedge\left(i^{*} \omega\right)^{n-1}$ is a volume form on $\mathcal{Z}$. Borman, Eliashberg and Murphy have shown that every almost contact manifold admits an overtwisted contact structure $\widetilde{\alpha}$ such that $d \widetilde{\alpha}$ is conformally equivalent to the original 2-form $i^{*} \omega$ [3]. It seems very unlikely that their construction could be made equivariant.

\section{The cohomology of toric origami manifolds}

In this section, we study the cohomology of toric origami manifolds. There are a number of cases where we can already deduce a number of facts about the cohomology of a toric origami manifold. We begin by reviewing these.

The first case is when the template graph is acyclic: this is the topic of our first paper [13]. In that case, the cohomology of $M$ is concentrated in even degrees and the 
equivariant cohomology is given by a GKM-type description as detailed in [13], or a Stanley-Reisner face ring [16, Theorem 7.7]. Furthermore, the ring structure on $H^{*}(M ; \mathbb{Z})$ can be determined completely from the discrete geometry of the orbit space, as described in [16, Corollary 7.8].

The second case where the cohomology ring is determined is when $M$ is prismatic. Then by Proposition 2-11, $M$ is homeomorphic to $Y \times \mathbb{T}^{2}$, for a toric symplectic manifold $Y$. Therefore the cohomology ring is determined by the Künneth formula, even over $\mathbb{Z}$ since the cohomology of each factor is torsion-free.

We now focus on the non-prismatic case, where we can obtain some partial results even for the cyclic case. We will use a Mayer-Vietoris sequence to obtain the Betti numbers of an arbitrary non-prismatic 4-dimensional toric origami manifold. We first note that in general,

$$
H^{0}(M ; \mathbb{Z}) \cong H^{2 n}(M ; \mathbb{Z})=\mathbb{Z}
$$

because $M$ is a connected $2 n$-dimensional manifold. Less trivially, $H^{2 n-1}(M ; \mathbb{Z}) \cong$ $H_{1}(M ; \mathbb{Z})$ is the abelianization of $\pi_{1}(M)$. By Theorem 2-14, it is thus $N / N_{X} \times \mathbb{Z}^{\ell}$, where $\ell=1+R-L$ is the number of linearly independent cycles in the template graph, which has $L$ vertices and $R$ edges. We also note that the universal coefficients theorem then guarantees that $H^{1}(M ; \mathbb{Z}) \cong \mathbb{Z}^{\ell}$ and that the torsion in $H^{2}(M ; \mathbb{Z})$ is precisely $N / N_{X}$.

We now proceed with our Mayer-Vietoris sequence. We enumerate the connected components $A_{1}, \ldots, A_{L}$ of $M \backslash Z$ and cover $M$ by open neighborhoods of each $A_{i}$. These open neighborhoods may be chosen so that they deformation retract to the $A_{i}$, and their intersections deformation retract onto components of $Z$. The Mayer-Vietoris sequence, with integer coefficients, is

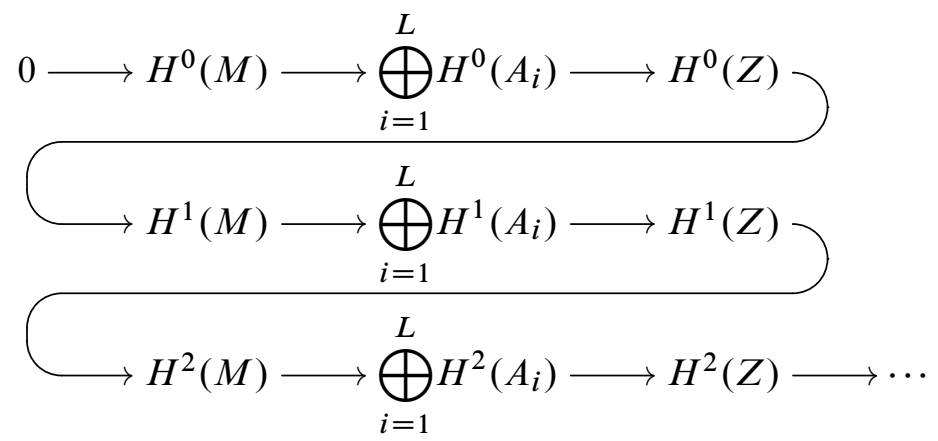

The techniques of Section 3 often enable us to calculate the ranks of the terms $\bigoplus_{i=1}^{L} H^{k}\left(A_{i}\right)$. As discussed in Section 4 , the connected components of $Z$ are $\mathbb{S}^{1}-$ bundles over compact toric symplectic manifolds of dimension $2 n-2$, and we may 
use our explicit description of the ordinary and equivariant Gysin sequences of this bundle to compute the Betti numbers of each connected component of $Z$. In some cases, we may perform each of these calculations explicitly. It may then be possible to determine the Betti numbers of $M$. In particular, when $2 n=4$, we can complete all of these steps. We will also include an example in dimension $2 n=6$.

Theorem 5-2 Let $M$ be a 4-dimensional toric origami manifold. If $M$ is prismatic, then it is homeomorphic to $\mathbb{S}^{2} \times \mathbb{T}^{2}$ and its Betti numbers are

$$
\beta^{0}(M)=\beta^{4}(M)=1 \quad \text { and } \quad \beta^{1}(M)=\beta^{2}(M)=\beta^{3}(M)=2 .
$$

If $M$ is non-prismatic, let $L$ be the number of vertices and $R$ the number of edges of its template graph, and $M^{\mathbb{T}}$ denote the set of (isolated) fixed points. Then

$$
\beta^{i}(M)= \begin{cases}1 & i=0,4, \\ 1+R-L & i=1,3, \\ \#\left(M^{\mathbb{T}}\right)+2 R-2 L & i=2 .\end{cases}
$$

In particular, in both cases the Euler characteristic is $\chi(M)=\#\left(M^{\mathbb{T}}\right)$.

Proof In the prismatic case, the result is a consequence of Proposition 2-11 and the Künneth formula. In this case, $\chi(M)=\#\left(M^{\mathbb{T}}\right)=0$.

We now turn to the non-prismatic case. Let $X=M / \mathbb{T}$ be the orbit space of $M$. The fixed points $M^{\mathbb{T}}$ correspond to vertices of $X$ (not to be confused with vertices of the template graph!).

We first consider the terms $\bigoplus_{i=1}^{L} H^{*}\left(A_{i}\right)$ in (5-1). We begin by noting that when $\operatorname{dim}(M)=4$, Theorem 3-12 determines all the Betti numbers of each piece $A_{i}$. Let $P$ be the number of prismatic $A_{i}$. Note that for 2-dimensional polytopes the number of facets (ie edges!) equals the number of vertices. A careful application of Theorem 3-12 now gives us

$$
\begin{array}{ll}
\sum_{i=1}^{N} \beta^{1}\left(A_{i}\right)=P, & \sum_{i=1}^{N} \beta^{2}\left(A_{i}\right)=\#\{\text { vertices of } X\}+2 R-2 L+P, \\
\sum_{i=1}^{N} \beta^{3}\left(A_{i}\right)=2 R-L, & \sum_{i=1}^{N} \beta^{4}\left(A_{i}\right)=0 .
\end{array}
$$

We now turn to the terms $H^{*}(Z)$ in (5-1). When $\operatorname{dim} M=2 n=4$, each $\mathcal{Z}$ is an $\mathbb{S}^{1}$-bundle over a toric symplectic 2-sphere, and is therefore diffeomorphic to $\mathbb{S}^{1} \times \mathbb{S}^{2}$, 
to $\mathbb{S}^{3}$ or to a 3 -dimensional lens space $\mathcal{L}=L(k ; 1)$. The Betti numbers of these spaces are

$$
\beta^{j}\left(\mathbb{S}^{1} \times \mathbb{S}^{2}\right)=\left\{\begin{array}{ll}
1 & \text { if } j=0,1,2,3, \\
0 & \text { otherwise }
\end{array} \quad \text { and } \quad \beta^{j}\left(\mathbb{S}^{3} \text { or } \mathcal{L}\right)= \begin{cases}1 & \text { if } j=0,3 \\
0 & \text { otherwise }\end{cases}\right.
$$

We do know that $\beta^{0}(M)=\beta^{4}(M)=1$ and $\beta^{1}(M)=\beta^{3}(M)=1+R-L$. Thus, the only group in the sequence (5-1) whose rank we do not know is $H^{2}(M)$. We proceed by dimension count. Let $Q$ be the number of connected components of $Z$ diffeomorphic to $\mathbb{S}^{1} \times \mathbb{S}^{2}$. Taking the alternating sum of the dimensions of the groups in the Mayer-Vietoris sequence (5-1), we have

$$
\begin{array}{r}
1-L+R-(1+R-L)+P-Q+\beta^{2}(M)-(\#\{\text { vertices of } X\}+2 R-2 L+P) \\
+Q-(1-R+L)+(2 R-L)-R+1=0
\end{array}
$$

if and only if

$$
\beta^{2}(M)=\#\{\text { vertices of } X\}+2 R-2 L,
$$

completing the proof.

Remark 5-3 An edge (1-dimensional face) of the orbit space $X$ of a toric origami manifold $M$ is either is a loop or has two end vertices. In the first case the edge is the moment image of a 2-torus, in the second it is the moment image of a sphere, with the end vertices being the image of the north and south poles of that sphere. As a consequence, $X$ can never have exactly one vertex, and $M$ can never have exactly one fixed point. Thus the Euler characteristic of a toric origami manifold cannot be equal to 1 .

The manifold $\mathbb{C P} \#\left(\mathbb{S}^{1} \times \mathbb{S}^{3}\right)$, made up of the building blocks mentioned in Remark 2-18, has Euler characteristic

$$
\chi\left(\mathbb{C} P^{2} \#\left(\mathbb{S}^{1} \times \mathbb{S}^{3}\right)\right)=\chi\left(\mathbb{C P}^{2}\right)+\chi\left(\mathbb{S}^{1} \times \mathbb{S}^{3}\right)-\chi\left(\mathbb{S}^{4}\right)=3+0-2=1
$$

and therefore does not admit a toric origami structure.

Remark 5-4 The second Betti number of a toric origami manifold bears a resemblance to that of a toric symplectic manifold. We have just seen that for a 4-dimensional toric origami manifold $M$, setting $\ell=1+R-L$,

$$
\beta^{2}(M)=\#\{\text { vertices in } M / \mathbb{T}\}-2+2 \ell .
$$

For a toric symplectic manifold $Y$ of dimension $2 n$,

$$
\beta^{2}(Y)=\#\{\text { facets in } Y / \mathbb{T}\}-n \text {. }
$$


In dimension 4, we can rewrite (5-5) as

$$
\beta^{2}(Y)=\#\{\text { vertices in } Y / \mathbb{T}\}-2 .
$$

Thus these two descriptions are the same, up to a correction for the rank $\ell$ of $\pi_{1}(M / \mathbb{T})$.
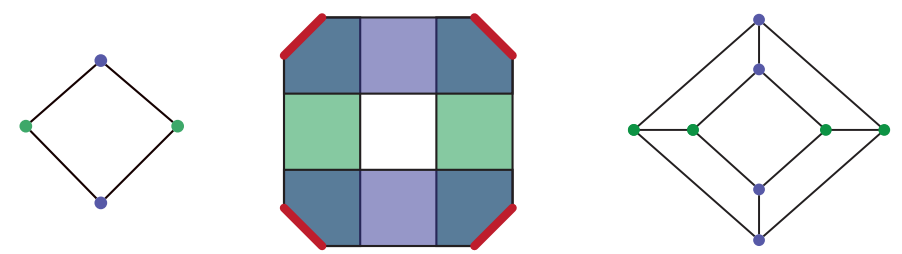

Figure 5.6: Left: the template graph of a toric origami manifold $M_{1}$. Center: the moment image of the toric origami manifold $M_{1}$. Right: the template graph of a toric origami manifold $M_{2}$ obtained from taking two copies of $M_{1}$ and gluing their orbit spaces along 4 pairs of non-folded facets.

Example 5-7 Let $M_{1}$ be the toric origami manifold described in Figure 5.6, left and center. This information completely determines the template and therefore the manifold. Its template graph has 4 vertices and 4 edges, and the manifold has 8 fixed points. Using Theorem 5-2 we conclude that the Betti numbers of this manifold are

$$
\beta^{0}\left(M_{1}\right)=\beta^{1}\left(M_{1}\right)=\beta^{3}\left(M_{1}\right)=\beta^{4}\left(M_{1}\right)=1 \quad \text { and } \quad \beta^{2}\left(M_{1}\right)=8 .
$$

The orbit space of $M_{1}$ has 4 non-folded facets, each corresponding to a symplectic 2-sphere embedded in $M_{1}$. Let $M_{2}$ be the toric origami manifold obtained by taking two copies of $M_{1}$ and gluing them together along each of the 4 pairs of symplectic 2 -spheres with the same moment image. The resulting template graph is on the righthand side of Figure 5.6 and has 8 vertices and 12 edges. The vertices and edges that appear in each of the two concentric square rings of this template graph correspond to the two copies of $M_{1}$, the remaining 4 edges in the template graph correspond to the new connected components of the fold. The manifold $M_{2}$ thus created has no fixed points. Using Theorem 5-2 we obtain its Betti numbers:

$$
\beta^{0}\left(M_{2}\right)=\beta^{4}\left(M_{2}\right)=1, \quad \beta^{1}\left(M_{2}\right)=\beta^{3}\left(M_{2}\right)=5, \quad \beta^{2}\left(M_{2}\right)=8 .
$$

Example 5-8 We now turn to a higher-dimensional example for which the computations are still tractable and for which we can obtain all the Betti numbers. Let $M$ be obtained from two copies of the manifold examined in Example 3-13, glued together along the two agreeing pairs of facets marked in red. 

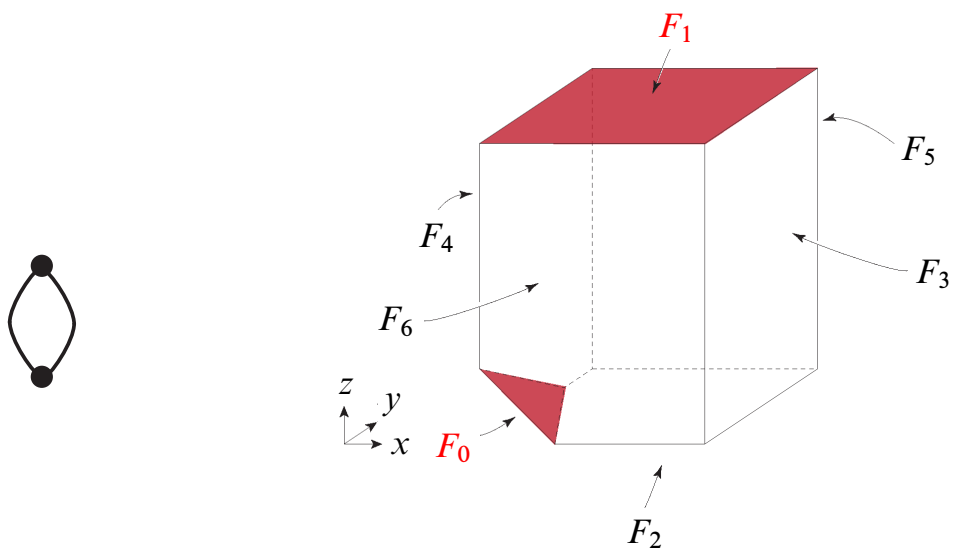

Figure 5.9: Left: the template graph of the manifold $M$. Right: each vertex of the template correponds to a copy of the toric symplectic manifold with moment image a truncated cube (the same as in Figure 3.14). One edge of the template graph corresponds to gluing together the pair of facets $F_{0}$, the other edge to gluing together the pair of facets $F_{1}$.

Most of the terms in the Mayer-Vietoris sequence with integer coefficients (5-1) are known, the $\bigoplus_{i=1}^{L} H^{*}\left(A_{i}\right)$ terms from Example 3-13 and the $H^{*}(Z)$ terms from direct computation. Indeed, $Z$ is the disjoint union $Z=\mathbb{S}^{5} \sqcup\left(\mathbb{S}^{2} \times \mathbb{S}^{2} \times \mathbb{S}^{1}\right)$, the first with moment image the facet $F_{0}$ and the second with moment image the facet $F_{1}$ in Figure 5.9, and therefore

$$
H^{k}(Z ; \mathbb{Z})= \begin{cases}\mathbb{Z}^{2} & \text { for } k=0,2,3,5 \\ \mathbb{Z} & \text { for } k=1,4\end{cases}
$$

Furthermore, we know that

$$
H^{0}(M ; \mathbb{Z})=H^{6}(M ; \mathbb{Z})=\mathbb{Z}
$$

because $M$ is a 6 -dimensional connected manifold and that

$$
H^{1}(M ; \mathbb{Z})=\mathbb{Z} \quad \text { and } \quad H^{5}(M ; \mathbb{Z})=\mathbb{Z}
$$

because $\pi_{1}(M)=\mathbb{Z}$. Taking an alternating sum of the ranks of the groups in the sequence (5-1), we obtain the remaining Betti numbers of $M$ :

$\beta^{0}(M)=\beta^{1}(M)=\beta^{3}(M)=\beta^{5}(M)=\beta^{6}(M)=1 \quad$ and $\quad \beta^{2}(M)=\beta^{4}(M)=2$. 


\section{References}

[1] MF Atiyah, Convexity and commuting Hamiltonians, Bull. London Math. Soc. 14 (1982) 1-15 MR642416

[2] M Ayzenberg, A Masuda, H Zeng, Cohomology of toric origami manifolds with acyclic proper faces (2014) arXiv:1407.0764

[3] MS Borman, Y Eliashberg, E Murphy, Existence and classification of overtwisted contact structures in all dimensions, preprint (2014) arXiv:1404.6157

[4] A Cannas da Silva, Lectures on symplectic geometry, Lecture Notes in Mathematics 1764, Springer, Berlin (2001) MR1853077

[5] A Cannas da Silva, V Guillemin, A R Pires, Symplectic origami, Int. Math. Res. Not. 2011 (2011) 4252-4293 MR2836021

[6] D A Cox, J B Little, H K Schenck, Toric varieties, Graduate Studies in Mathematics 124, Amer. Math. Soc. (2011) MR2810322

[7] V I Danilov, The geometry of toric varieties, Uspekhi Mat. Nauk 33 (1978) 85-134 MR495499 In Russian; translated in Russian Math. Surveys 33 (1978) 97-154

[8] T Delzant, Hamiltoniens périodiques et images convexes de l'application moment, Bull. Soc. Math. France 116 (1988) 315-339 MR984900

[9] W Fulton, Introduction to toric varieties, Annals of Mathematics Studies 131, Princeton Univ. Press (1993) MR1234037

[10] R L Graham, M Grötschel, L Lovász (editors), Handbook of combinatorics, Elsevier, Amsterdam (1995) MR1373655

[11] V Guillemin, S Sternberg, Geometric quantization and multiplicities of group representations, Invent. Math. 67 (1982) 515-538 MR664118

[12] A Hatcher, Algebraic topology, Cambridge Univ. Press (2002) MR1867354

[13] T S Holm, A R Pires, The topology of toric origami manifolds, Math. Res. Lett. 20 (2013) 885-906 MR3207359

[14] S Luo, Cohomology rings of good contact toric manifolds (2012) arXiv: 1012.2146

[15] W S Massey, Algebraic topology: An introduction, Graduate Texts in Mathematics 56, Springer, New York (1977) MR0448331

[16] M Masuda, T Panov, On the cohomology of torus manifolds, Osaka J. Math. 43 (2006) 711-746 MR2283418

[17] M Masuda, S Park, Toric origami manifolds and multi-fans, Proc. Steklov Math. Inst. 286 (2014) 308-323

[18] J Møller, From singular chains to Alexander duality, lecture notes (2008) Available at http://www.math.ku.dk/ moller/f03/algtop/notes/homology.pdf 
[19] P Orlik, F Raymond, Actions of the torus on 4-manifolds II, Topology 13 (1974) 89-112 MR0348779

[20] P S Pao, The topological structure of 4-manifolds with effective torus actions I, Trans. Amer. Math. Soc. 227 (1977) 279-317 MR0431231

Department of Mathematics, Cornell University 571 Malott Hall, Ithaca, NY 14850-4201, USA

Department of Mathematics, Fordham University - Lincoln Center 113 W 60th St., Room 813, New York, NY 10023-7414, USA tsh@math.cornell.edu, apissarrapires@fordham.edu

Received: 21 July 2014 Revised: 3 December 2014 
